\title{
OPEN Respiration and brain neural dynamics associated with interval timing during odor fear learning in rats
}

\author{
Maryne Dupin ${ }^{1 凶}$, Samuel Garcia ${ }^{1}$, Belkacem Messaoudi ${ }^{1}$, Valérie Doyère ${ }^{2,3}$ \& \\ Anne-Marie Mouly ${ }^{1 凶}$
}

In fear conditioning, where a conditioned stimulus predicts the arrival of an aversive stimulus, the animal encodes the time interval between the two stimuli. Here we monitored respiration to visualize anticipatory behavioral responses in an odor fear conditioning in rats, while recording theta $(5-15 \mathrm{~Hz})$ and gamma (40-80 Hz) brain oscillatory activities in the medial prefrontal cortex (mPFC), basolateral amygdala (BLA), dorsomedial striatum (DMS) and olfactory piriform cortex (PIR). We investigated the temporal patterns of respiration frequency and of theta and gamma activity power during the odorshock interval, comparing two interval durations. We found that akin to respiration patterns, theta temporal curves were modulated by the duration of the odor-shock interval in the four recording sites, and respected scalar property in MPFC and DMS. In contrast, gamma temporal curves were modulated by the interval duration only in the MPFC, and in a manner that did not respect scalar property. This suggests a preferential role for theta rhythm in interval timing. In addition, our data bring the novel idea that the respiratory rhythm might take part in the setting of theta activity dynamics related to timing.

Interval timing refers to the ability to time intervals ranging from seconds to minutes and guides fundamental animal behaviors like the anticipation of rewarding or aversive events. The tasks classically used in the literature to assess interval timing in animals, necessitate numerous training sessions and involve a motor response from the animal ${ }^{1}$. Yet some studies show that in associative learning, animals learn to time the arrival of reinforcement from the outset of conditioning ${ }^{2-7}$ and such temporal encoding has been suggested to be a fundamental component of associative learning ${ }^{8}$. However, the neurobiological basis of interval timing in Pavlovian associative learning remain poorly understood, due in part to the paucity of studies designed for its investigation'.

In a previous study using odor fear conditioning in rats, we showed that, when using an appropriate index, namely the respiratory rate, interval timing can be inferred from the animal's behavior after a few training trials ${ }^{7}$. More specifically, the animal's respiratory rate was monitored in this paradigm where an initially neutral odor signals the arrival of an aversive mild foot-shock at a fixed time interval. We showed that after a few odor-shock pairings, the respiratory frequency curve presented a temporal pattern that was linked to the duration of the interval to be timed, in a manner that respected scalar property, a hallmark of interval timing, i.e. the error magnitude in estimating a duration was proportional to the duration to be timed ${ }^{10}$.

Based on these findings, in the present study we investigated the neural network dynamics occurring during the odor-shock interval in odor fear conditioning in rats. Although the neural mechanisms underlying timing remain largely unknown, several studies in the literature reported dynamically changing patterns of activity that contain information about elapsed time since a given stimulus. This property has been found in multiple brain areas including the dorsal striatum and the prefrontal cortex ${ }^{11,12}$. More specifically, electrophysiological recording of neurons in the medial prefrontal cortex (mPFC) and the dorsomedial striatum (DMS) in rats engaged in interval timing, have shown that ramping activity, a monotonic change in neuronal firing rate across time, is observed throughout frontostriatal ensembles. Importantly, DMS ramping neurons were shown to synchronize with $\mathrm{mPFC} 4-\mathrm{Hz}$ activity and inactivation of the mPFC impaired interval timing and attenuated ramping

\footnotetext{
${ }^{1}$ Lyon Neuroscience Research Center, INSERM U1028, CNRS UMR 5292, Université Lyon 1, 69366 Lyon, France. 'Université Paris-Saclay, CNRS, Institut des Neurosciences Paris-Saclay, 91190 Gif-sur-Yvette, France. ${ }^{3} \mathrm{NYU}$ Child Study Center Department of Child and Adolescent Psychiatry, New York University Langone School of Medicine, New York, USA. ${ }^{\circledR}$ email: maryne.dupin@live.fr; annemarie.mouly@cnrs.fr
} 


\section{A- Training protocol}

Group 20s

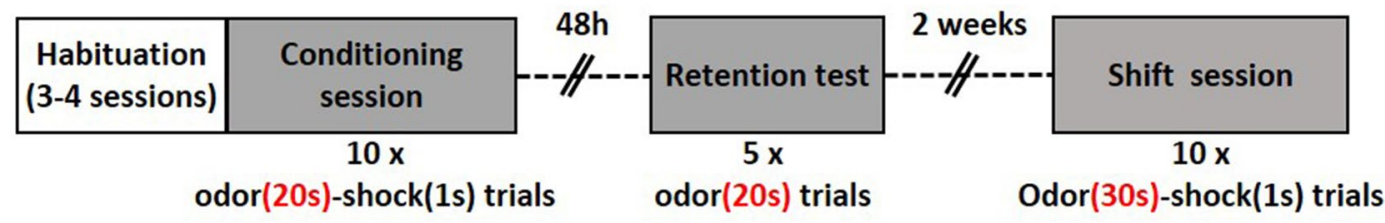

\section{Group 30s}

\begin{tabular}{|c|c|}
\hline $\begin{array}{c}\text { Habituation } \\
\text { (3-4 sessions) }\end{array}$ & $\begin{array}{c}\text { Conditioning } \\
\text { session }\end{array}$ \\
\hline & odor(30s)-shock(1s) trials
\end{tabular}

\section{B- Data recording and analysis}

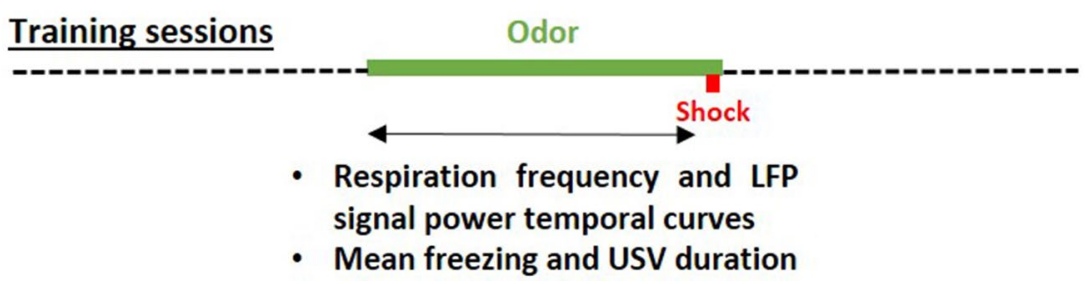

Figure 1. (A) Training protocol. Group $20 \mathrm{~s}$ : the animals were trained with 10 Odor-Shock pairings using a $20 \mathrm{~s}$ Odor-Shock interval (Conditioning session). $48 \mathrm{~h}$ later, they were tested for their retention of the learning (Retention test) with 5 presentations of the learned odor. Two weeks later they were trained with 10 OdorShock pairings using a 30 s Odor-Shock interval (Shift session). Group 30 s: The animals were trained with 10 Odor-Shock pairings using a 30 s Odor-Shock interval (Conditioning session). (B) Data recording and analysis. Training sessions: in the two groups, during the odor-shock interval, local field potentials (LFP), respiration, ultrasonic vocalizations and freezing behavior were recorded, synchronized and analyzed as described in the "Methods".

activity in the $\mathrm{DMS}^{13}$. In addition, optogenetic stimulation of mPFC axon terminals modulated time-related ramping activity of medium spiny neurons in the striatum and improved interval-timing behavior ${ }^{14}$, suggesting that the mPFC exerts top-down control of temporal processing in the DMS. On the other hand, recent studies using aversive conditioning suggest that the amygdala also plays a role in interval timing , $^{7,9,15,16}$ and regulates physiological correlates of plasticity in the striatum, thanks to direct amygdala projections to the striatum ${ }^{16}$. In addition, evidence of time encoding has been found in different sensory cortices like the primary visual cortex ${ }^{17,18}$ and the olfactory piriform cortex ${ }^{19}$. Therefore, in the present study, rats were implanted with chronic electrodes in four brain areas: the mPFC, DMS, basolateral amygdala (BLA) and piriform cortex (PIR). The animals were trained in an odor fear conditioning paradigm while their respiration was measured, together with brain oscillatory activities in the targeted network. We focused on variations in the theta $(5-15 \mathrm{~Hz})$ and gamma $(40-80 \mathrm{~Hz})$ bands that have been shown to reflect temporal processing ${ }^{16,20-22}$.

Our prediction was that, if a brain area is involved in timing the odor-shock interval, temporal curves of oscillatory activity power during that interval should (1) build up after a few odor-shock pairings in parallel to respiratory frequency temporal curves, and (2) be modulated by the duration of the interval in a manner that respects scalar property.

\section{Results}

Respiratory frequency is a good index of interval timing providing the level of stress is not too high. In a previous study ${ }^{7}$ we showed that respiration is a good index of interval timing in non-implanted animals. We assessed if this was still true for implanted animals, in which the level of stress could be higher and might constrain the range of the respiratory response.

The time course of respiratory frequency during the odor-shock interval in Group $20 \mathrm{~s}$ was compared between the Conditioning session where a $20 \mathrm{~s}$ interval was used (see protocol in Fig. 1), and the Shift session for which the interval was shifted to $30 \mathrm{~s}$ (Fig. 2A). The ANOVA revealed no significant effect of Interval, but a significant effect of Time $\left(\mathrm{F}_{17,340}=11.43, \mathrm{p}<0.0001\right)$ and a significant Interval $\times$ Time interaction $\left(\mathrm{F}_{17,340}=3.029, \mathrm{p}<0.0001\right)$. 


\section{A- Respiratory frequency time course}

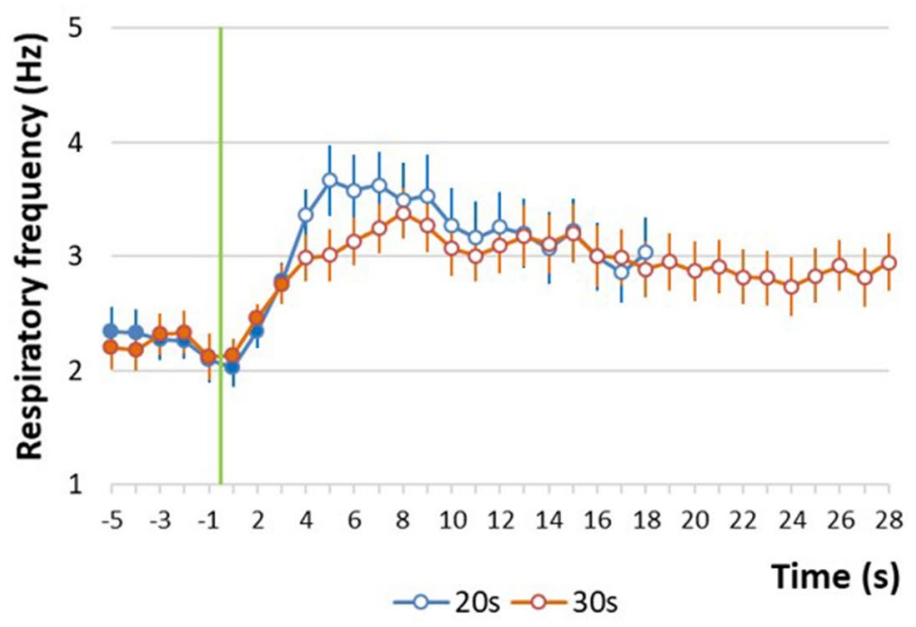

\section{B- Good Timers versus Bad Timers}
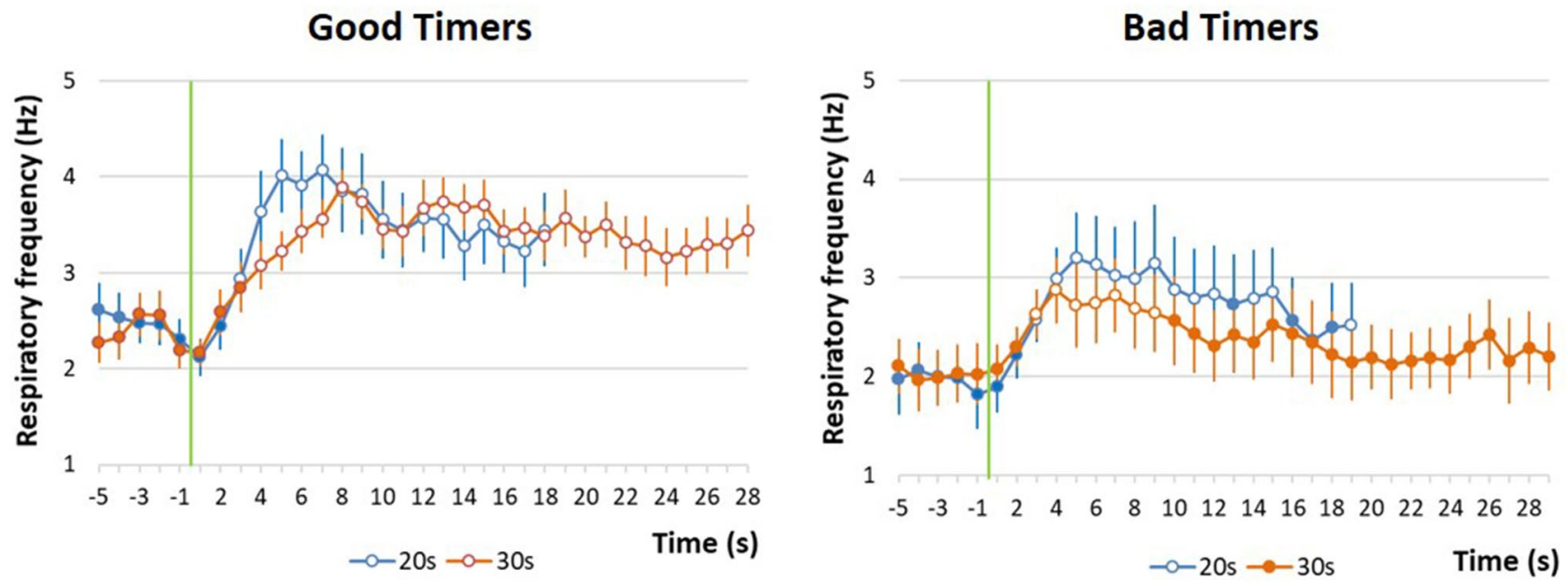

Figure 2. (A) Respiratory frequency time course in Group $20 \mathrm{~s}$. The time course of respiratory frequency is represented with a 1-s bin precision, from $5 \mathrm{~s}$ before odor onset (green vertical line) to shock arrival which occurred either at $20 \mathrm{~s}$ (Conditioning session, in blue) or at $30 \mathrm{~s}$ (Shift session 2, in orange). (B) Good Timers versus Bad Timers subgroups. In Good Timers subgroup, the temporal patterns of respiratory frequency were modulated by the duration of the odor-shock interval, whereas in Bad Timers subgroup, they were not. Open circles on each curve signal the values that are significantly different from the baseline $(\mathrm{p} \leq 0.05)$.

As depicted on Fig. 2A, shifting from a $20 \mathrm{~s}$ interval to a $30 \mathrm{~s}$ interval resulted in an increase of the mean latency to the peak respiratory frequency on the temporal curve ( $5 \mathrm{~s}$ for $20 \mathrm{~s}$ interval vs $8 \mathrm{~s}$ for $30 \mathrm{~s}$ interval).

However, we noticed a strong inter-individual variability among animals concerning the shift of the temporal curve when the interval duration was changed to $30 \mathrm{~s}$, with animals presenting clearly shifted temporal curves for $30 \mathrm{~s}$ intervals, and animals exhibiting similar curves for both intervals. Because we previously showed that the shift in respiration temporal pattern was a good indicator of interval duration encoding ${ }^{7}$, we reasoned that animals that did not exhibit this shift should be analyzed separately from those presenting a shift. To do so, we measured for each animal the time at which the respiratory frequency peaked for the $20 \mathrm{~s}$ - and $30 \mathrm{~s}$-interval temporal curves and calculated the difference between the two obtained values. When this value was $\geq 2 \mathrm{~s}$, the animal was classified as a Good Timer, the remaining animals were classified as Bad Timers. This led to two subgroups: subgroup Good Timers $(n=12$, mean latency difference $=6.08 \pm 0.96 \mathrm{~s})$ and subgroup Bad Timers $(\mathrm{n}=9$, mean latency difference $=-2.22 \pm 1.00 \mathrm{~s})$. The temporal curves obtained in these two subgroups are presented in Fig. 2B. In the Good Timers subgroup (Fig. 2B, left), the ANOVA confirmed a significant effect of Time $\left(\mathrm{F}_{17,187}=16.31, \mathrm{p}<0.0001\right)$ and a significant Interval $\times$ Time interaction $\left(\mathrm{F}_{17,187}=3.09, \mathrm{p}<0.0001\right)$, with no significant effect of Interval. A significant interaction between interval duration and elapsed time indicates that the time course of the respiratory frequency changes is affected by the duration of the odor-shock interval. In the Bad Timers subgroup (Fig. 2B, right), the ANOVA revealed a significant effect of Time $\left(\mathrm{F}_{17,136}=3.59, \mathrm{p}<0.0001\right)$ but no significant effect of Interval and no significant Interval $\times$ Time interaction. Interestingly, the difference between Good Timers and Bad Timers is mainly due to the temporal patterns exhibited during the Shift session. 
Indeed the temporal patterns displayed by both subgroups during the Conditioning session ( $20 \mathrm{~s}$ interval) were similar (Group $\times$ Time interaction: $\mathrm{F}_{17,323}=0.94, \mathrm{p}=0.53$ ), while those displayed during the Shift session $(30 \mathrm{~s}$ interval) differed significantly (Group $\times$ Time interaction: $\mathrm{F}_{27,513}=3.07, \mathrm{p}<0.001$ ).

The temporal patterns observed in Good Timers subgroup suggest that the Odor-Shock interval was timed according to the scalar rule ${ }^{10}$. In order to assess scalar timing quantitatively, the time axis for the 30-s data was multiplicatively rescaled so that the 18 time-bins during the interval for both groups represented the same proportions of elapsed time from odor onset to shock presentation. The scalar timing rule predicts superior superposition of the functions in relative time, compared to no rescaling. Supplementary Fig. S1 shows raw and rescaled mean curves for both 20 - and 30-s conditions, for Good Timers and Bad Timers subgroups. Superposition was indexed by eta-squared $\left(\eta^{2}\right)$, a measure of the proportion of variance accounted for by the mean of the two functions $s^{23}$. When superposition is perfect, $\eta^{2}$ is at its maximum value of 1 . For Good Timers, $\eta^{2}$ was greater under the multiplicative transform $\left(\eta^{2}=0.93\right.$, Supplementary Fig. S1A, right $)$ than under no rescaling $\left(\eta^{2}=0.88\right.$, Supplementary Fig. S1A, left). In contrast, for Bad Timers subgroup, $\eta^{2}$ was greater under no rescaling $\left(\eta^{2}=0.73\right.$, Supplementary Fig. S1B, left) than under the multiplicative transform $\left(\eta^{2}=0.50\right.$, Supplementary Fig. S1B, right).

We next evaluated to what extent the timing abilities assessed through respiration could be related to different levels of fear exhibited by the animals. To do so, we compared the mean amount of freezing exhibited by the animals in the Good Timers and Bad Timers subgroups during the Odor-Shock interval in the Conditioning and Shift sessions (Fig. 3A, Left). The ANOVA revealed no significant effect of Subgroup, Training session, and Training session $\times$ Subgroup interaction. We then compared the number of USV emitted by the animals in the two subgroups during the Odor-Shock interval for the two training sessions (Fig. 3A, right). The ANOVA revealed a significant effect of Subgroup $\left(\mathrm{F}_{1,16}=8.59, \mathrm{p}=0.010\right)$ and Training session $\left(\mathrm{F}_{1,16}=7.91, \mathrm{p}=0.013\right)$, and no significant Training session $\times$ Subgroup interaction. Further pairwise comparisons showed that Bad Timers animals emitted more USV than Good Timers during both training sessions ( $\mathrm{p}=0.012$ for the $20 \mathrm{~s}$ interval session; $\mathrm{p}=0.015$ for the $30 \mathrm{~s}$ interval session).

Finally, we assessed whether the two subgroups presented different levels of learned fear during the $48 \mathrm{~h}$ retention test (Fig. 3B). In each subgroup, we compared the amounts of freezing observed before odor introduction and during odor. The ANOVA revealed a significant effect of Period (Pre-Odor vs Odor; $\mathrm{F}_{1,18}=35.56, \mathrm{p}<0.0001$ ) but no significant effect for Subgroup, nor for Period $\times$ Subgroup interaction. In both subgroups, the amount of freezing was significantly higher $(\mathrm{p}<0.005)$ in presence of the learned odor than before its delivery suggesting similar levels of fear memory in Bad Timers and Good Timers animals.

Together, these data show that the temporal pattern observed in respiration rate in the Good Timers subgroup reflects the learning of Odor-Shock interval duration, and confirm our previous finding that rats can learn the expected time of shock delivery in an odor fear conditioning task within 10 conditioning trials ${ }^{7}$. The ability to express temporal behavior related to interval duration, at least when looking at the respiratory rate, seems to depend on the animal's fear response: the higher the USV amount, the lower the possibility for respiration to exhibit different temporal patterns related to interval duration. Finally, this difference in timing abilities seems to have no impact on the strength of the fear memory as assessed through odor-elicited freezing $48 \mathrm{~h}$ later.

In the next analyses, we assessed whether the differences observed in timing expression in Bad Timers and Good Timers, based on their temporal pattern of respiratory frequency, were associated with similar differences in oscillatory activity temporal patterns.

Changes in oscillatory activity in the theta (5-15 Hz) band. We assessed whether, akin to respiratory frequency, the temporal patterns of theta activity power were modulated by the duration of the Odor-Shock interval.

As explained in the "Methods", after elimination of artifacted electrophysiological signals, the number of animals per recording site were the following: BLA, $n=17$; CPF, $n=20$; PIR, $n=20$; DMS, $n=19$ for Group $20 \mathrm{~s}$; BLA, $n=6$; CPF, $n=8$; PIR, $n=8$; DMS, $n=9$ for Group 30 s.

The temporal patterns of theta activity power during the Odor-Shock interval in Group 20 s were compared between the Conditioning session where a $20 \mathrm{~s}$ interval was used, and the Shift session for which the interval was shifted to $30 \mathrm{~s}$. Figure 4 presents the temporal curves obtained in each of the four recording sites for the Good Timers (left side) and Bad Timers (right side) subgroups. The ANOVA carried out in the Good Timers subgroup revealed no significant effect of Interval, but for all four structures a significant effect of Time $\left(m P F C: F_{17,187}=6.56, p<0.0001 ; B_{1}:_{17,119}=6.77, p<0.0001 ;\right.$ PIR: $F_{17,187}=4.15, p<0.0001 ;$ DMS: $F_{17,187}=6.24$, $\mathrm{p}<0.0001$ ), and a significant IntervalxTime interaction (mPFC: $\mathrm{F}_{17,187}=2.66, \mathrm{p}<0.0001 ; \mathrm{BLA}: \mathrm{F}_{17,119}=5.14$, $\mathrm{p}<0.0001$; PIR: $\mathrm{F}_{17,187}=3.47, \mathrm{p}<0.0001$; DMS: $\left.\mathrm{F}_{17,187}=4.26, \mathrm{p}<0.0001\right)$. In contrast, in the Bad Timers subgroup (Fig. 4, right panels), theta activity power showed almost no change during the interval compared to baseline in the four recording sites (no significant effect of Time or Interval), and only the mPFC showed a significant Interval $\times$ Time interaction $\left(\mathrm{F}_{17,119}=1.72, \mathrm{p}=0.047\right)$.

These data suggest that in Good Timers, the time course of theta activity power was modulated by the duration of the interval to be timed. In order to assess scalar timing quantitatively, the time axis for the 30-s data was multiplicatively rescaled and the $\eta^{2}$ was calculated for each recording site (Supplementary Fig. S2). In mPFC and DMS, $\eta^{2}$ was greater under the multiplicative transform (Supplementary Fig. S2, right) than under no rescaling (Supplementary Fig. S2, left), while the reverse was observed for BLA and PIR.

These data show that in Good Timers animals, akin to what is observed for respiration frequency, the time course of theta activity power was related to the learned duration of Odor-Shock interval in the four recording sites, and in a manner that respected scalar property in the MPFC and the DMS. 


\section{A- Freezing and USV during the Odor-Shock interval in the Conditioning and Shift sessions}
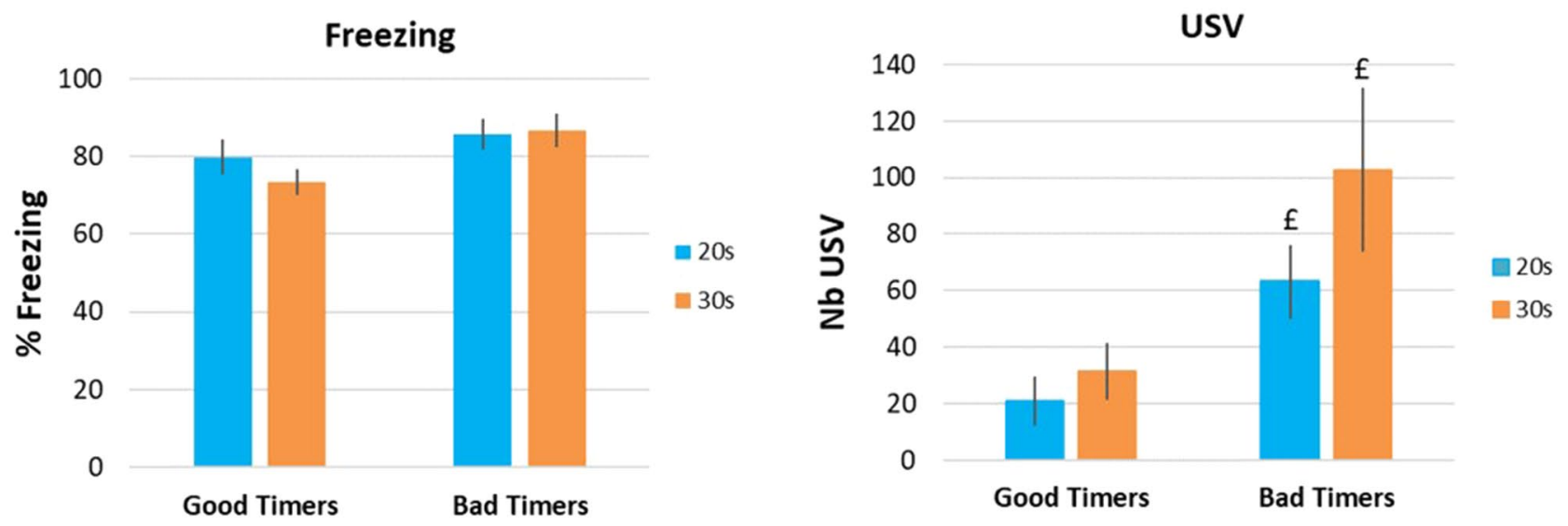

\section{B- Freezing response during the Retention test}

$$
100
$$

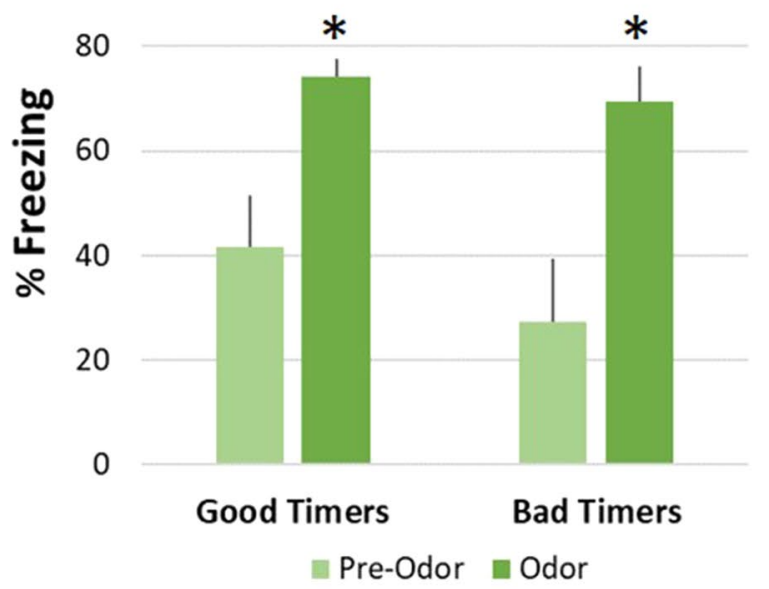

Figure 3. (A) Freezing and ultrasonic vocalizations (USV) during the two training sessions, in Good Timers and Bad Timers subgroups. Freezing amount and number of USVs were measured during the $20 \mathrm{~s}$ (Conditioning session, in blue) and $30 \mathrm{~s}$ (Shift session, in orange) odor-shock intervals throughout the training session. ${ }^{E}$ Significant between group difference $(\mathrm{p} \leq 0.05)$. (B) Freezing amount during the Retention test. Good Timers and Bad Timers subgroups showed similar levels of freezing during the Retention test. ${ }^{*}$ Significant within group difference with pre-odor period $(\mathrm{p}<0.005)$.

Changes in oscillatory activity in the gamma $(40-80 \mathrm{~Hz})$ band. Similar comparisons were made for the temporal pattern of gamma activity during the Odor-Shock interval. Figure 5 presents the temporal curves obtained in the four recording sites in the Good Timers (Left side) and Bad Timers (right side) subgroups. The ANOVA carried out in the Good Timers subgroup revealed no significant effect of Interval, but a significant effect of Time in mPFC $\left(\mathrm{F}_{17,187}=4.73, \mathrm{p}<0.0001\right)$, PIR $\left(\mathrm{F}_{17,187}=2.57, \mathrm{p}=0.002\right)$ and DMS $\left(\mathrm{F}_{17,187}=3.65, \mathrm{p}<0.0001\right)$, and a significant Interval $\times$ Time interaction only in $\mathrm{mPFC}\left(\mathrm{F}_{17,187}=2.51, \mathrm{p}=0.001\right)$. In the Bad Timers subgroup, the ANOVA revealed a significant effect of Time in $\operatorname{mPFC}\left(\mathrm{F}_{17,119}=2.24, \mathrm{p}=0.006\right)$, PIR $\left(\mathrm{F}_{17,119}=1.95, \mathrm{p}=0.02\right)$ and DMS $\left(\mathrm{F}_{17,102}=2.22, \mathrm{p}=0.007\right)$, but no significant effect of Interval nor Interval $\times$ Time interaction in the four recording sites.

Therefore, in both Good Timers and Bad Timers, gamma power increased significantly during the interval compared to baseline in the mPFC, DMS and PIR, but not in the BLA. When looking at the temporal patterns, the only structure in which the time course of gamma activity power was affected by the duration of the interval (i.e. showing an Interval $\times$ Time interaction) was the $\mathrm{mPFC}$ in the Good Timers subgroup. To assess scalar timing, the time axis for the 30-s data was multiplicatively rescaled and the $\eta^{2}$ was calculated (Supplementary Fig. S3). $\eta^{2}$ was greater under no rescaling (Supplementary Fig. S3, left) than under the multiplicative transform (Supplementary Fig. S3, right), suggesting gamma activity temporal pattern in MPFC does not solely reflect the learning of Odor-Shock interval duration. 


\section{Theta}

Good Timers

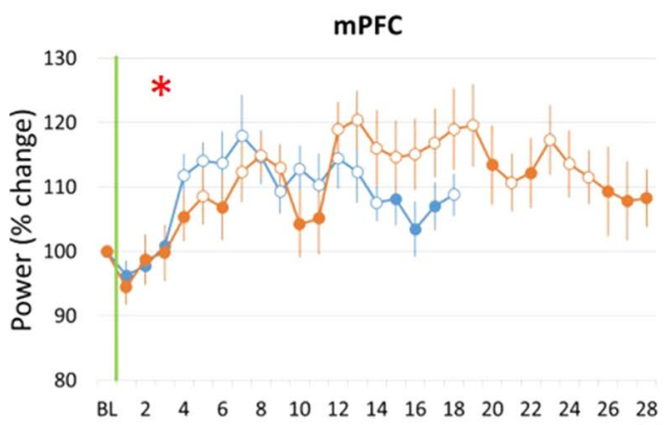

Times (s)

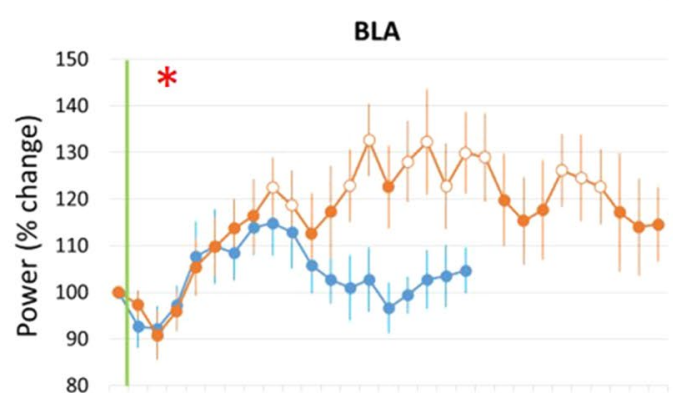

$\begin{array}{lllllllllllllll}\text { BL } & 2 & 4 & 6 & 8 & 10 & 12 & 14 & 16 & 18 & 20 & 22 & 24 & 26 & 28\end{array}$

Time (s)

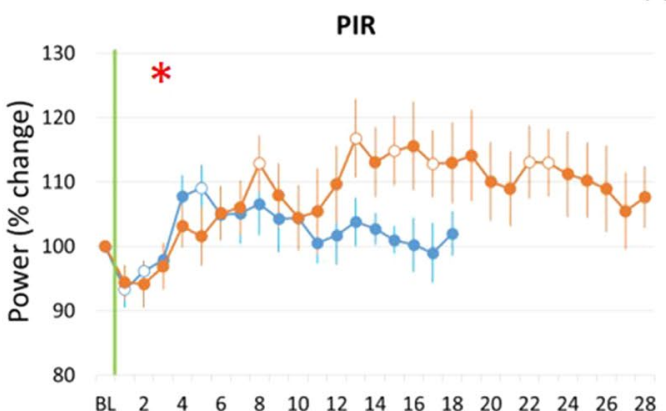

Time (s)

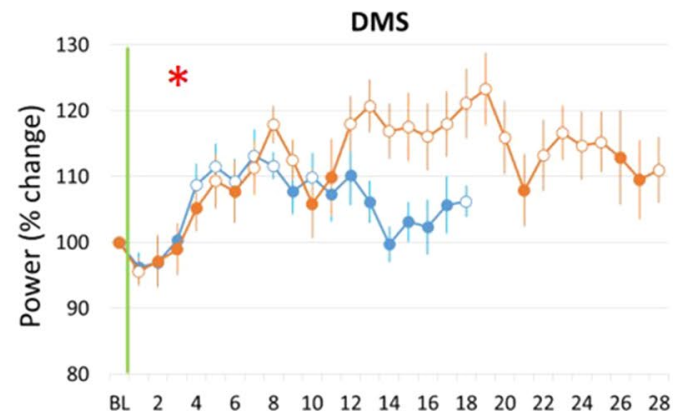

Time (s)
Bad Timers

mPFC

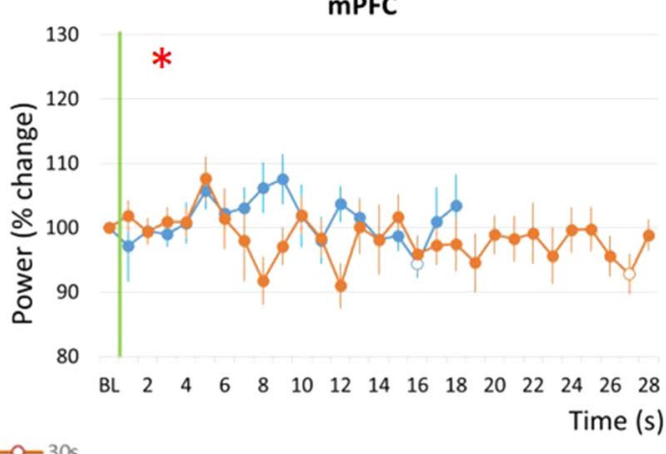

BLA
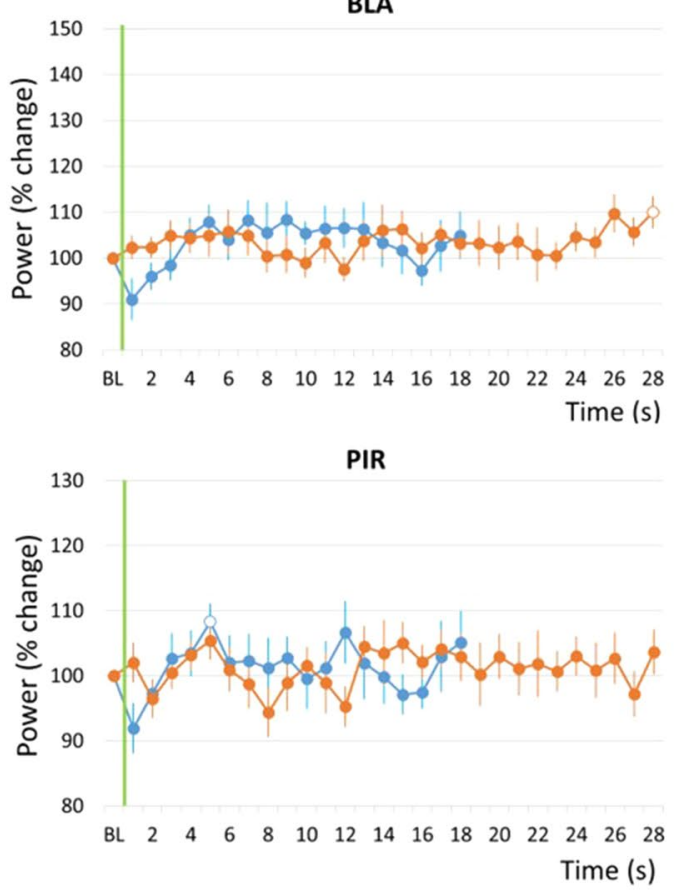

DMS

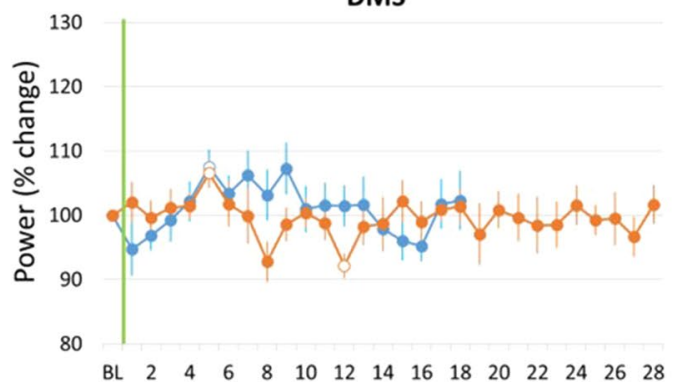

Time (s)

Figure 4. Theta $(5-15 \mathrm{~Hz})$ band mean power variations during the odor-shock interval in the Good Timers (left side) and Bad Timers (right side) subgroups in the four recording sites. $m P F C$ medial prefrontal cortex, $B L A$ basolateral amygdala, PIR olfactory piriform cortex, DMS dorso-medial striatum. For each graph, theta power was expressed as percent change from the baseline (BL), and represented from odor onset (green vertical line) to shock arrival which occurs either at $20 \mathrm{~s}$ (Conditioning session, in blue) or at $30 \mathrm{~s}$ (Shift session, in orange). The red asterisk in the upper left corner of a graph signals a significant time $\times$ interval duration interaction. Open circles on each curve signal the values that are significantly different from the baseline $(\mathrm{p} \leq 0.05)$. 


\section{Gamma}

Good Timers

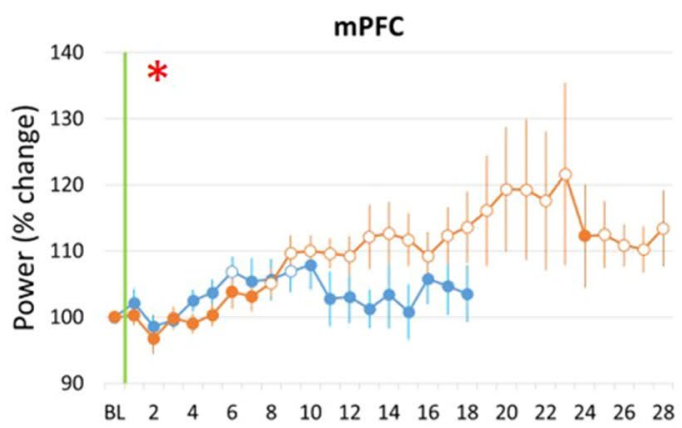

Time (s)

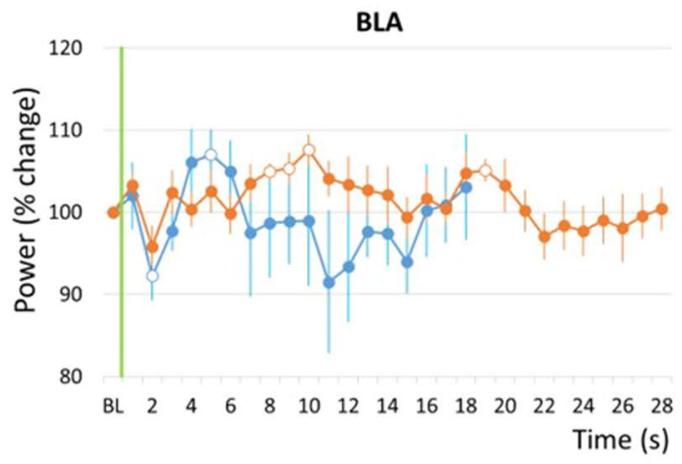

PIR

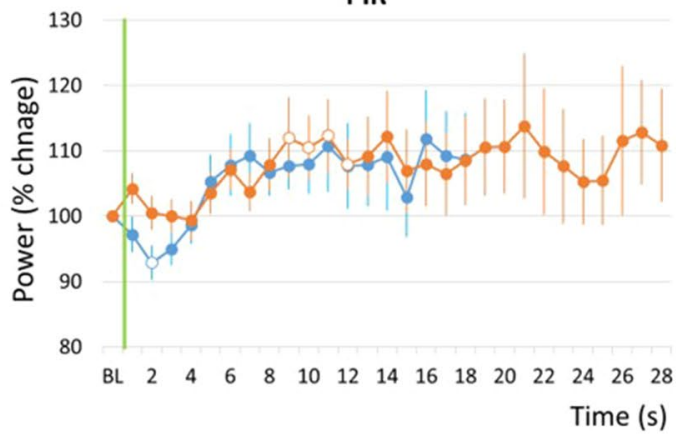

DMS

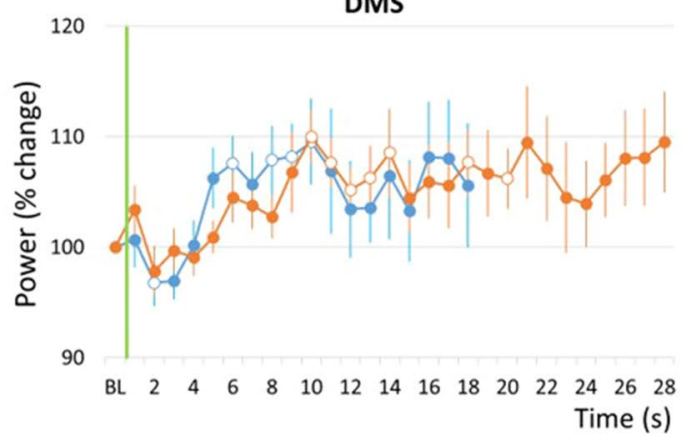

Bad Timers
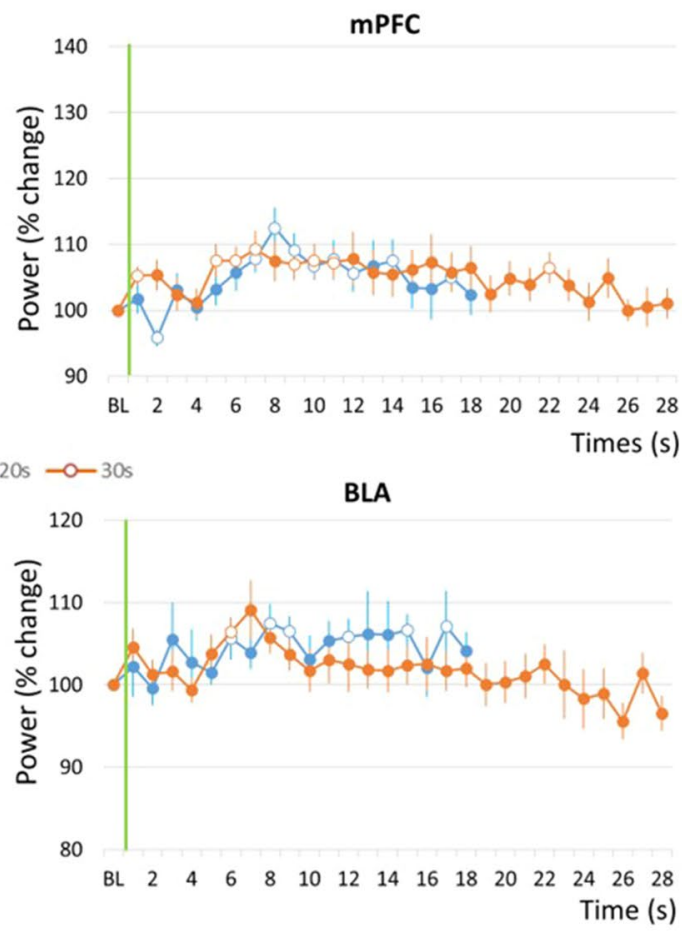

PIR

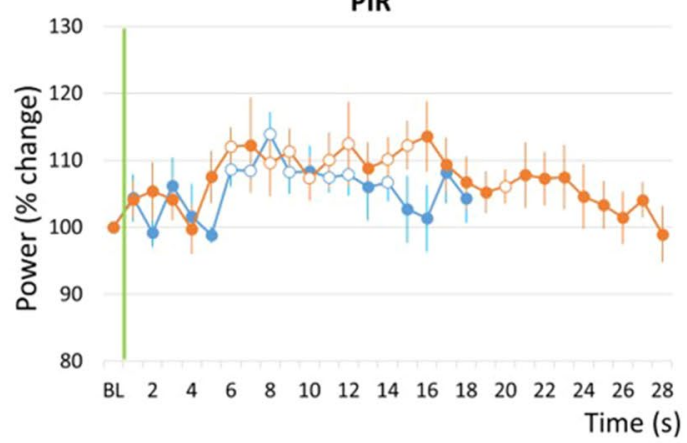

DMS

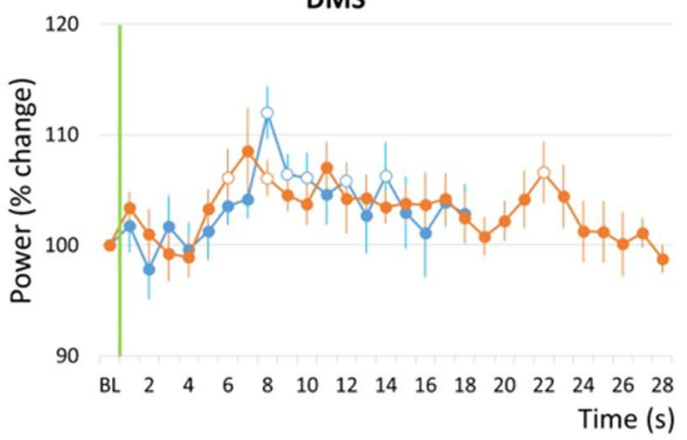

Figure 5. Gamma (40-80 Hz) band mean power variations during the odor-shock interval in the Good Timers (left side) and Bad Timers (right side) subgroups in the four recording sites. $m P F C$ medial prefrontal cortex, $B L A$ basolateral amygdala, PIR olfactory piriform cortex, DMS dorso-medial striatum. For each graph, theta power was expressed as percent change from the baseline (BL), from the odor onset (green vertical line) to shock arrival which occurs either at $20 \mathrm{~s}$ (Conditioning session, in blue) or at $30 \mathrm{~s}$ (Shift session, in orange). The red asterisk in the upper left corner of a graph signals a significant time $\times$ interval duration interaction. Open circles on each curve signal the values that are significantly different from the baseline $(\mathrm{p} \leq 0.05)$. 
Correlation between respiration temporal curves and theta/gamma power temporal curves. Together the data presented so far suggest that in Good Timers, there might be a correlation between the time course of respiration frequency curve and that of theta activity power curves. To assess this quantitatively, we performed two kinds of correlation analysis. First, we measured for each animal the time of the peak respiratory frequency and the time of the peak theta activity power during the Conditioning session and calculated the correlation index between these values (Fig. 6A). This analysis showed significant positive correlation for BLA $\left(R_{8}=0.85, p<0.01\right)$, PIR $\left(R_{12}=0.63, p<0.02\right)$ and DMS $\left(R_{12}=0.61, p=0.02\right)$, and a tendency for mPFC $\left(R_{12}=0.50\right)$. We performed the same analysis for gamma activity and found no significant correlation with respiration (mPFC: $\mathrm{R}_{12}=0.26$, ns; $B L A: \mathrm{R}_{8}=0.21$, ns; PIR: $\mathrm{R}_{12}=0.24$, ns; $\mathrm{DMS}$ : $\mathrm{R}_{12}=-0.09$, ns) (Fig. $6 \mathrm{~B}$ ).

For the second correlation analysis, we calculated the difference between the times of the peak respiratory frequency during the Conditioning (20 s interval) and Shift (30 s interval) sessions: Peak $30 \mathrm{~s}-\mathrm{Peak} 20 \mathrm{~s}$ (Shift R, Fig. 7A). Similarly, for each recording site, we calculated the difference between the times of the peak theta activity power during the Conditioning and Shift sessions: Peak $30 \mathrm{~s}-$ Peak $20 \mathrm{~s}$ (Shift T, Fig. 7B, upper part). Then we calculated the correlation between Shift R and Shift T (Fig. 7B, lower part). The data show a significant positive correlation between the two values for the $\operatorname{mPFC}\left(\mathrm{R}_{12}=0.62, \mathrm{p}<0.02\right)$, but not for the other sites (BLA: $\mathrm{R}_{8}=0.43$, ns; PIR: $\mathrm{R}_{12}=-0.49$, ns; DMS: $\mathrm{R}_{12}=0.32$, ns). The same analysis was carried out for gamma activity in $\mathrm{mPFC}$ and showed no significant correlation (Shift G, Fig. 7C, R12 =0.39, ns).

In summary, in the four recorded structures, the time course of theta activity power correlates with the time course of respiratory frequency. In addition, in $\mathrm{mPFC}$, the shift in theta activity power temporal curves observed when the interval duration was changed, was positively correlated with the shift in respiratory frequency temporal curve. These correlations were not observed when considering gamma activity power.

Interval timing in independent groups. In the experiments carried out so far, the same animals received two successive training sessions, a Conditioning session with a 20 s odor-shock interval, and a Shift session with a $30 \mathrm{~s}$ interval. This allowed us to compare the temporal curves within the same rats, thus discounting interindividual variability. However, the data obtained during the Shift session might have been partly confounded by the previously experienced Conditioning session. To assess this point, a small group of animals was trained in a single session using a $30 \mathrm{~s}$ odor-shock interval (Group $30 \mathrm{~s}$ ). We compared the temporal curves obtained in Good Timers during the Conditioning session of Group $20 \mathrm{~s}$ to those of the single session of Group $30 \mathrm{~s}$. The only difference between the two groups was the duration of the odor-shock interval with an equivalent level of training.

Figure 8A (left part) presents the temporal curves obtained for respiratory frequency in the two groups. The ANOVA revealed no significant effect of Group, but a significant effect of Time $\left(\mathrm{F}_{17,238}=5.39, \mathrm{p}<0.001\right)$ and a significant Time $\times$ Group interaction $\left(\mathrm{F}_{17,238}=2.01, \mathrm{p}=0.012\right)$. When the time axis for the $30 \mathrm{~s}$ data was multiplicatively rescaled to assess scalar timing (Fig. 8A, right part), the $\eta^{2}$ index increased from 0.56 (no rescaling) to 0.62 (multiplicative transform).

Figure $8 \mathrm{~B}$, upper left part, presents the temporal curves obtained for theta activity power in mPFC in the two groups. The ANOVA carried out in the four recording sites revealed no effect of Group, but a significant effect of Time in $\mathrm{mPFC}\left(\mathrm{F}_{17,238}=2.44, \mathrm{p}=0.002\right)$, and a significant Time $\times$ Group interaction in $\mathrm{mPFC}\left(\mathrm{F}_{17,238}=2.32\right.$, $\mathrm{p}=0.003)$, BLA $\left(\mathrm{F}_{17,153}=2.18, \mathrm{p}=0.007\right)$ and DMS $\left(\mathrm{F}_{17,238}=2.11, \mathrm{p}=0.01\right)$ (Supplementary Fig. S4, left part). When the time axis for the 30 -s data was multiplicatively rescaled to assess scalar timing, $\eta^{2}$ was greater under the multiplicative transform than under no rescaling in mPFC, BLA and DMS (Fig. 8B upper right part, and Supplementary Fig. S4 right part).

Figure $8 \mathrm{~B}$, lower left part, presents the temporal curves obtained for gamma activity power in $\mathrm{mPFC}$ in the two groups. The ANOVA carried out in the four recording sites (Supplementary Fig. S5, left part) revealed no effect of Group, but a significant effect of Time in $\operatorname{mPFC}\left(\mathrm{F}_{17,238}=4.21, \mathrm{p}<0.0001\right)$, PIR $\left(\mathrm{F}_{17,221}=2.12, \mathrm{p}=0.007\right)$ and DMS $\left(\mathrm{F}_{17,238}=3.45, \mathrm{p}<0.0001\right)$, and a significant Time $\times$ Group interaction in $\operatorname{mPFC}\left(\mathrm{F}_{17,221}=3.30, \mathrm{p}<0.0001\right)$ and DMS $\left(\mathrm{F}_{17,238}=2.45, \mathrm{p}=0.001\right)$. When the time axis for the $30 \mathrm{~s}$ data was multiplicatively rescaled to assess scalar timing, $\eta^{2}$ was greater under the multiplicative transform than under no rescaling in mPFC and DMS (Fig. 8B, lower right part, and Supplementary Fig. S5 right part).

In summary, for the respiration and theta temporal curves, parallel training with either $20 \mathrm{~s}$ or $30 \mathrm{~s}$ odorshock interval duration in different animals brought globally the same kind of data as when animals were trained successively to the two intervals. Concerning gamma activity, parallel training revealed a modulation of activity power temporal curves by the interval's duration in MPFC and DMS in a manner that respected scalar property.

\section{Discussion}

The present study is the first to correlate brain neural dynamics associated with behavioral expression of interval timing at early stages of Pavlovian aversive conditioning. This was made possible thanks to the use of a sensitive behavioral index, respiration, which allowed getting a readout of the animal's interval timing ability without training the animal in a long and complex operant behavioral task. Here we show that in a successive training paradigm where the initially learned odor-shock interval duration is shifted to a new one, animals exhibiting good timing performances as assessed through respiration rate, also show temporal patterns of theta activity power modulated by the interval duration in the four recording sites, and those measured in mPFC and DMS followed the scalar property. In addition, the temporal patterns of theta activity in the mPFC faithfully followed the temporal patterns of respiration. In contrast, temporal patterns of gamma activity power modulated by the interval duration were observed only in $\mathrm{MPFC}$, and they failed to respect scalar property (see a summary of the data on Table 1, upper part). 


\section{A- Theta}
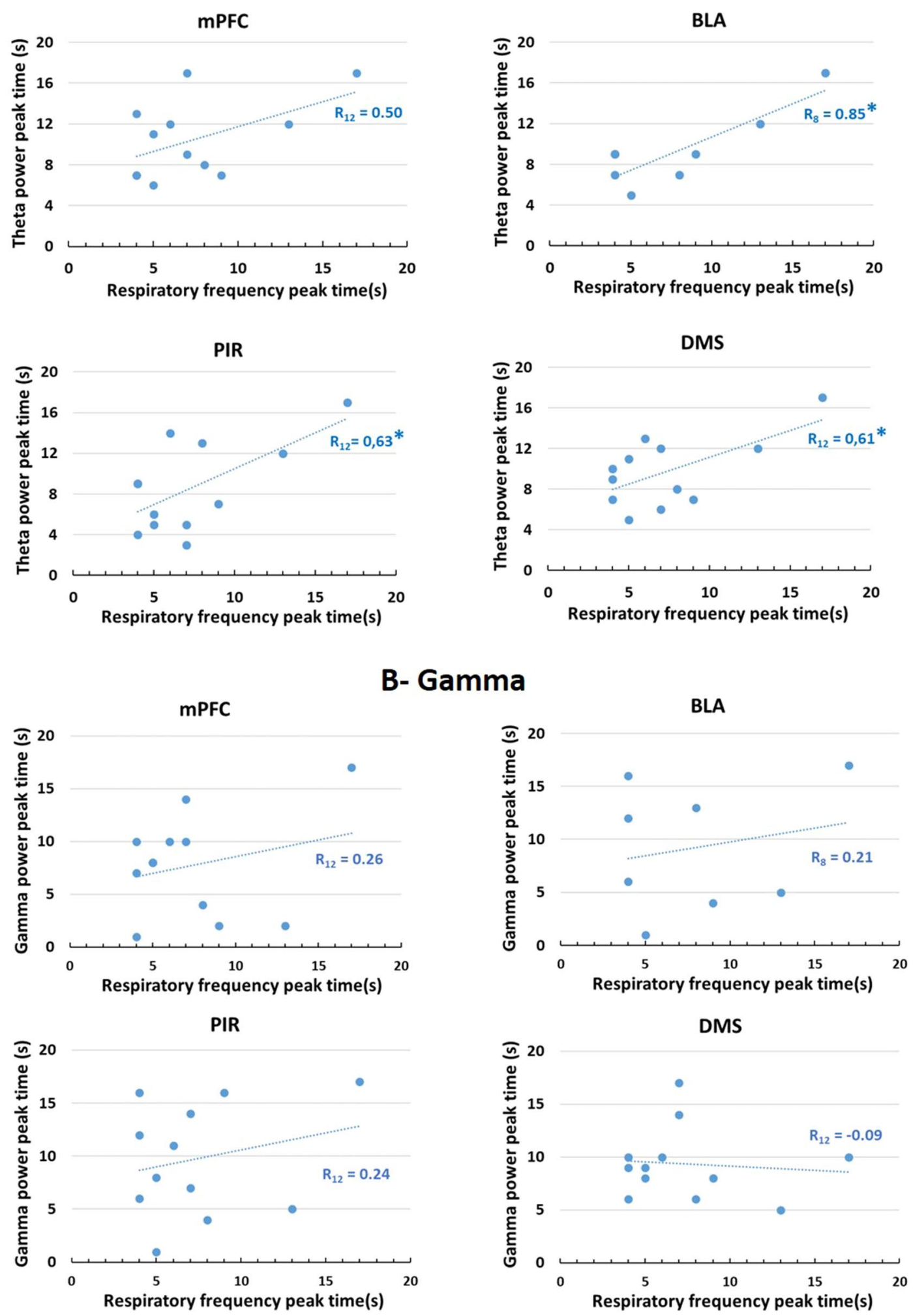

Figure 6. (A) Correlation between the time of the peak respiratory frequency and the time of the peak theta activity power in the four recording sites during the Conditioning session. $m P F C$ medial prefrontal cortex, $B L A$ basolateral amygdala, $P I R$ olfactory piriform cortex, DMS dorso-medial striatum. *Significant correlation index, $\mathrm{p} \leq 0.05$. (B) Correlation between the time of the peak respiratory frequency and the time of the peak gamma activity power in the four recording sites during the Conditioning session. 


\section{A- Respiration}

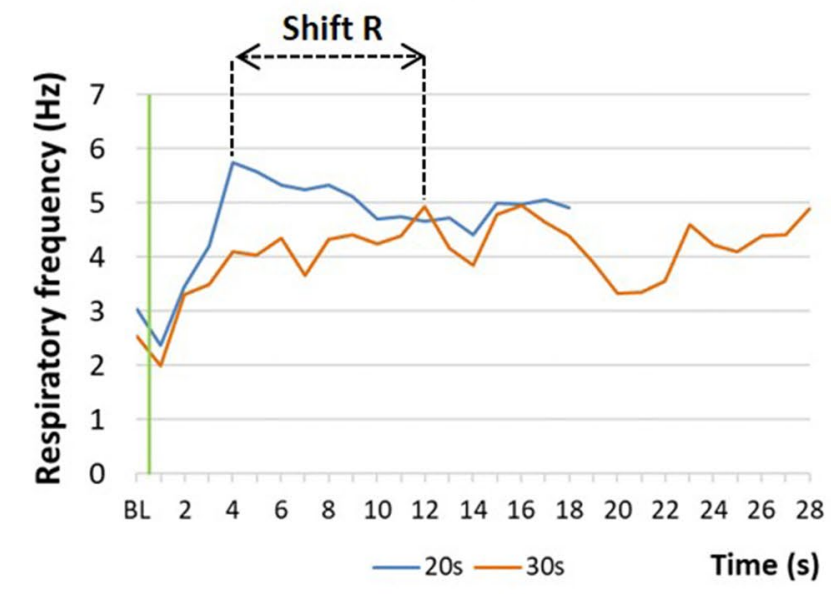

\section{B- Theta (mPFC)}

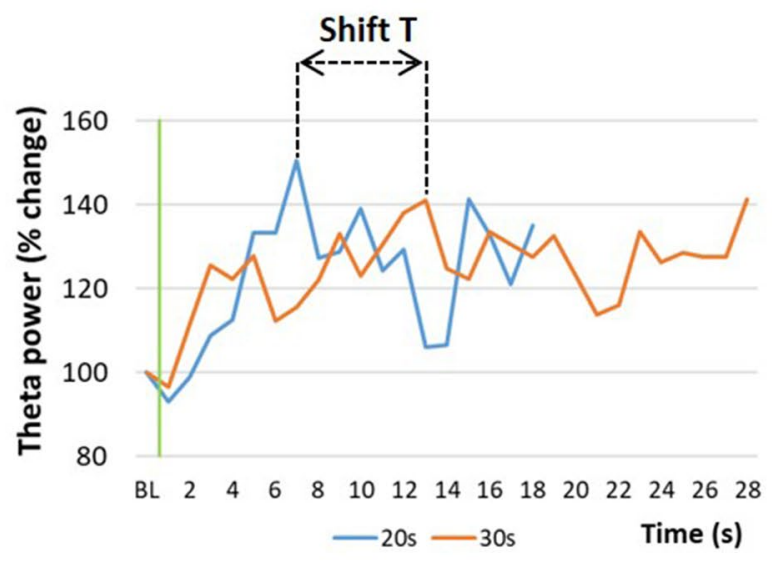

15

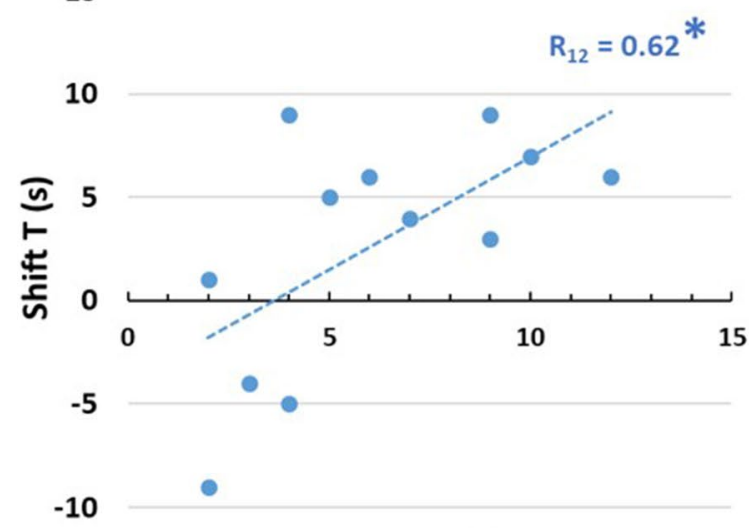

Shift R (s)
C- Gamma (mPFC)

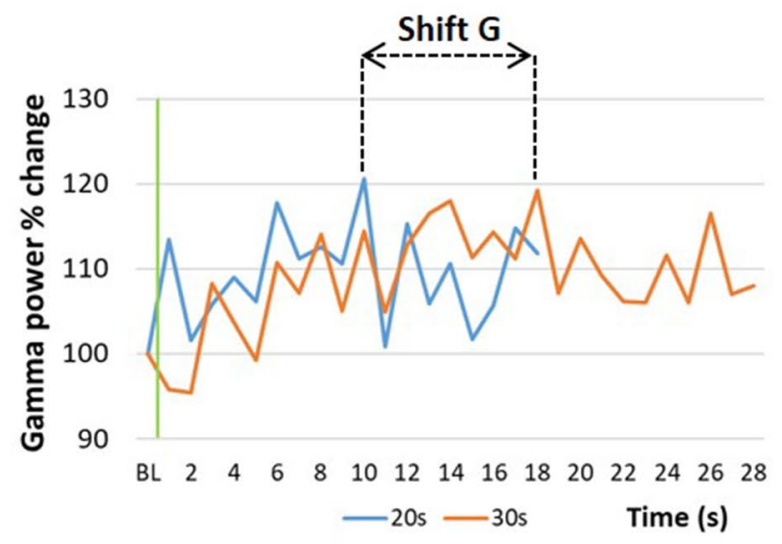

15

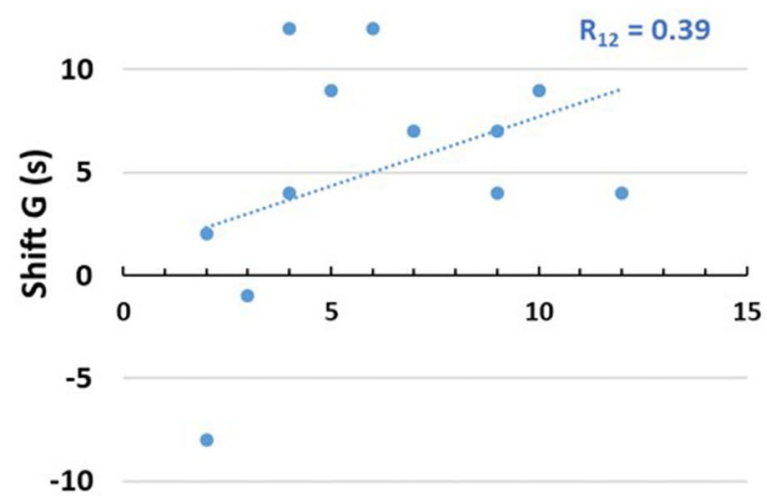

Shift R (s)

Figure 7. Correlation between the shift in respiration temporal curves and theta/gamma power temporal curves. (A) The time of the peak respiratory frequency during the Conditioning session (20 s interval) and the Shift session ( $30 \mathrm{~s}$ intervals) was measured and the difference between the two values was used as the Shift R index. (B) Upper graph: For each recording site, the time of the peak theta activity power during the Conditioning session (20 s interval) and the Shift session (30 s intervals) was measured and the difference between the two values was used as the Shift $\mathrm{T}$ index. The graph illustrates data from the mPFC: medial prefrontal cortex. Lower graph: the correlation between Shift $\mathrm{R}$ and Shift $\mathrm{T}$ indices was calculated. ${ }^{*}$ Significant correlation index, $\mathrm{p} \leq 0.05$. (C) Upper graph: For each recording site (example of the $\mathrm{mPFC}$ ), the time of the peak gamma activity power during the Conditioning session ( $20 \mathrm{~s}$ interval) and the Shift session (30 s intervals) was measured and the difference between the two values was used as the Shift G index. Lower graph: the correlation between Shift R and Shift G indices was calculated. 


\section{A- Parallel training, Respiratory frequency}
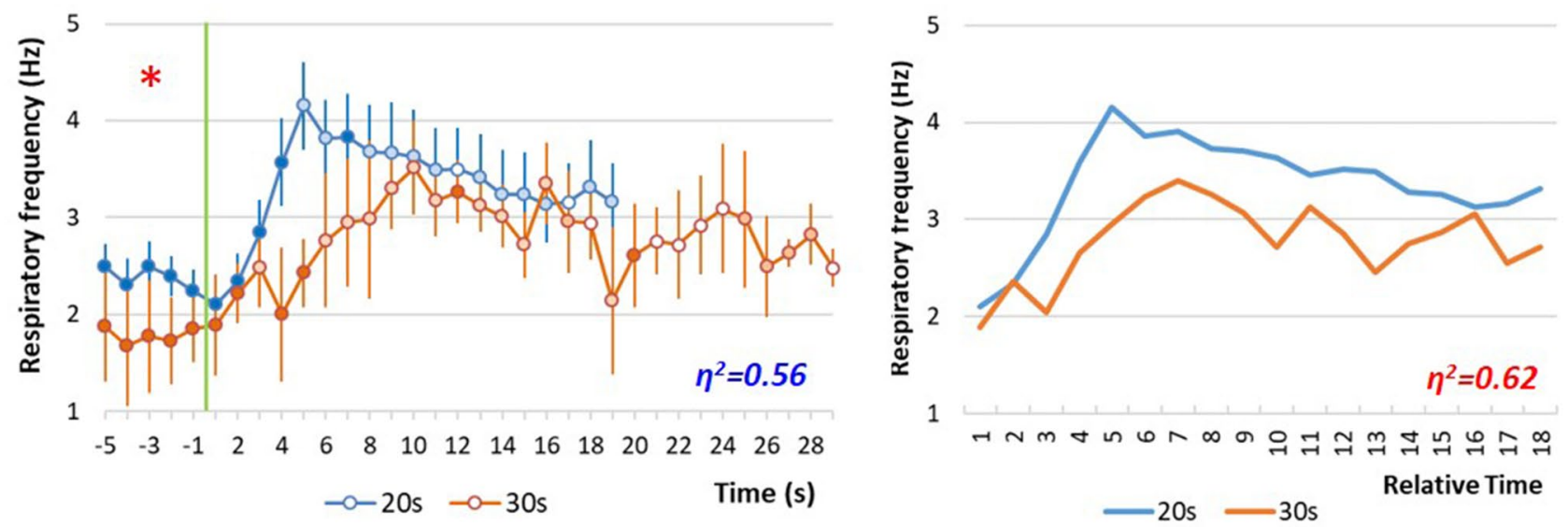

\section{Theta $(5-15 \mathrm{~Hz})$}

\section{B- Parallel training, LFP signal power}
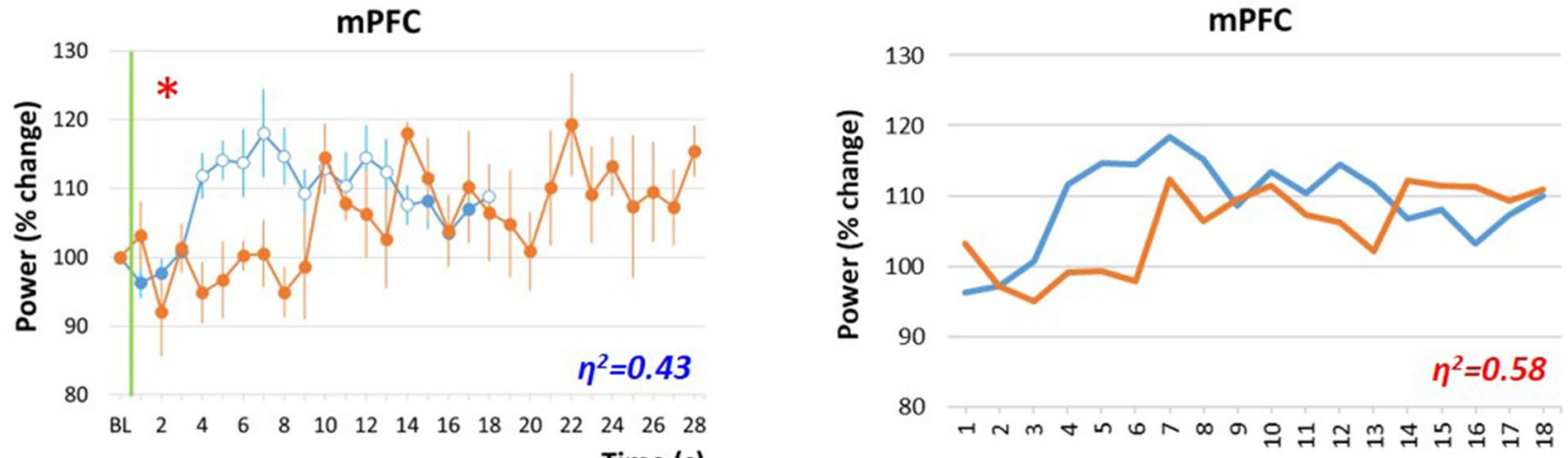

Time (s)

Relative Time

$$
-20 \text { - }-30 \text { s }
$$

\section{Gamma $(40-80 \mathrm{~Hz})$}

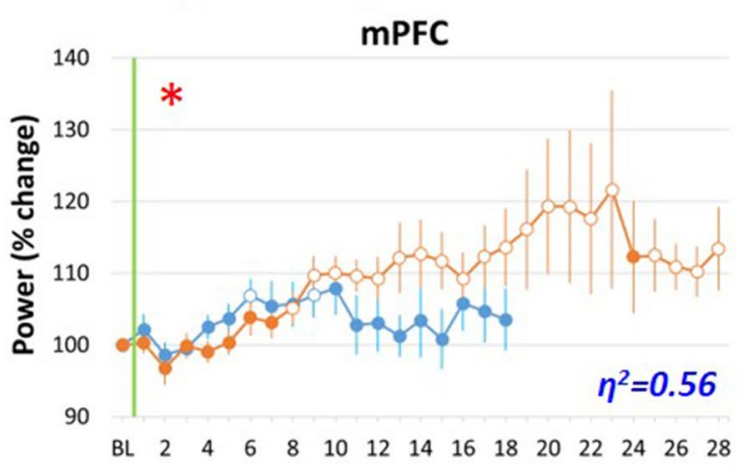

Time (s)

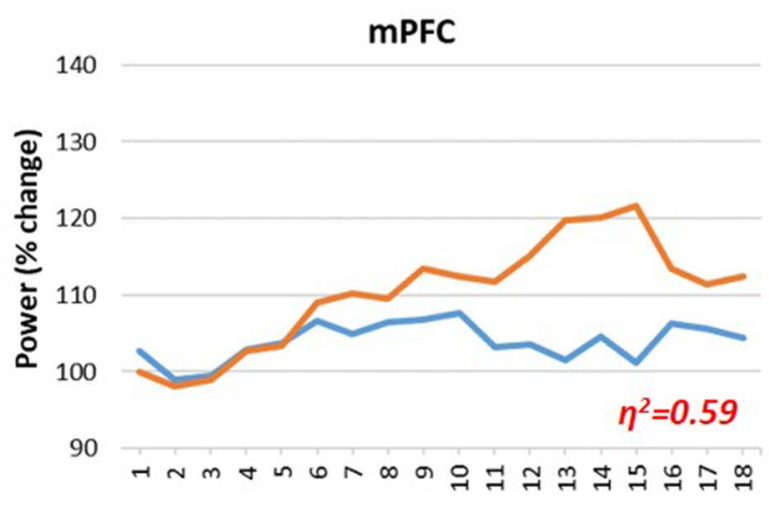

Relative Time

Figure 8. Behavioral and electrophysiological comparison in parallel training condition. (A) Respiratory frequency curves. Left side, raw data: the temporal pattern of respiratory frequency is represented with a 1-s bin precision, from $5 \mathrm{~s}$ before odor onset (green vertical line) to shock arrival which occurs either at $20 \mathrm{~s}$ (Group $20 \mathrm{~s}$, in blue) or at $30 \mathrm{~s}$ (Group $30 \mathrm{~s}$, in orange). Right side, rescaled curves: the time axis for the 30-s data (in orange) was multiplicatively rescaled. Superposition between the two curves was indexed by eta-square $\left(\eta^{2}\right)$ indicated in the bottom right of each graph, the highest values being highlighted in red. (B) Local field potential (LFP) signal power in the mPFC (medial prefrontal cortex), in the theta $(5-15 \mathrm{~Hz}$ ) and gamma $(40-80 \mathrm{~Hz})$ bands. Left side, raw data: theta and gamma power was expressed as percent change from the baseline (BL), and represented from odor onset (green vertical line) to shock arrival which occurs either at $20 \mathrm{~s}$ (Group $20 \mathrm{~s}$, in blue) or at $30 \mathrm{~s}$ (Group $30 \mathrm{~s}$, in orange). Right side, rescaled curves. The red asterisk in the upper left corner of a graph signals a significant time $\times$ interval duration interaction. Open circles on each curve signal the values that are significantly different from the baseline $(\mathrm{p}<0.05)$. Superposition between the two curves was indexed by eta-square $\left(\eta^{2}\right)$ indicated in the bottom right of each graph, the highest values being highlighted in red. 


\begin{tabular}{|c|c|c|c|c|}
\hline & \multicolumn{2}{|l|}{ Theta } & \multicolumn{2}{|l|}{ Gamma } \\
\hline & Interval $\times$ Time & Scalar property & Interval $\times$ Time & Scalar property \\
\hline \multicolumn{5}{|c|}{ Successive training } \\
\hline $\mathrm{mPFC}$ & $x$ & $x$ & $x$ & \\
\hline BLA & $x$ & & & \\
\hline PIR & $x$ & & & \\
\hline DMS & $x$ & $x$ & & \\
\hline \multicolumn{5}{|c|}{ Parallel training } \\
\hline $\mathrm{mPFC}$ & $x$ & $x$ & $x$ & $x$ \\
\hline BLA & $x$ & $x$ & & \\
\hline \multicolumn{5}{|l|}{ PIR } \\
\hline DMS & $x$ & $x$ & $x$ & $x$ \\
\hline
\end{tabular}

Table 1. Summary of the data obtained for theta and gamma activity power in Good Timers animals in the two experimental paradigms (Successive training and parallel training) and in the four recording sites, for the criteria relevant to interval timing, ie significant Interval $\times$ Time interaction and respect of scalar property. $m P F C$ medial prefrontal cortex, BLA basolateral amygdala, PIR olfactory piriform cortex, DMS dorso-medial striatum.

The present work highlights that respiratory rhythm can be used as a reliable index of interval timing providing that the level of stress of the animal is not too high. Indeed, we could distinguish two subgroups of animals based on their individual temporal pattern of respiratory frequency during the odor-shock interval, and its modification following the shift to a new interval duration. Good Timers presented temporal patterns that were modulated by the duration of the interval in a way that respected scalar property. In contrast, Bad Timers showed changes in respiratory frequency during the interval, but these changes were not modulated by the duration of the interval.

At the behavioral level, all the animals except two (out of 30) produced USV s albeit at different rates. This is in contrast to what is usually observed in non-implanted animals, where the ratio of vocalizing animals is generally around $50 \%$ for the same foot-shock parameters ${ }^{7,24,25}$. The reason for this high rate of USV vocalizing animals in the present study might be related to their housing condition, as animals implanted with electrodes were housed individually. It has been shown that single housing increases anxiety and biological markers of stress as compared to social housing ${ }^{26,27}$ and the amount of USV emission is enhanced when the level of anxiety is increased ${ }^{25}$.

Interestingly, Bad Timers showed more USV emissions than Good Timers during the two training sessions. Bad Timers were initially selected based on their respiration curves that were not affected by the change in interval duration, and it happened that they were also those presenting the highest rates of USV. The question then arises as to why rats emitting more USV exhibit poor timing abilities as assessed through respiration. A first explanation is that a drawback of choosing respiration rate as an index of timing is that any factor (other than timed anticipation) affecting respiration will indirectly affect its temporal pattern. USV emission strongly decreases respiratory frequency and constraints it's range of variations ${ }^{24,28,29}$ thus possibly blurring the temporal pattern of the respiration curve. A second explanation might be related to the fact that the higher amounts of USV in Bad Timers are a marker of higher levels of anxiety. Indeed, It is well known from the existing literature on $22 \mathrm{kHz}$ USV in rats, that there is a great inter-individual variability concerning the emission of USV in an aversive context, with some animals producing no USV at all, while other animals vocalize almost continuously during the entire recording session ${ }^{25}$. Importantly, such inter-individual variability has been related to differences in anxiety levels. Indeed, more anxious rats (as assessed through the elevated plus maze) were shown to be more likely to vocalize than less anxious animals ${ }^{30}$. Thus Bad Timers in our study, might be more anxious animals. A few studies have assessed the impact of fear on temporal behavior in animals. Among them, studies using a peak interval procedure ${ }^{31-33}$ or a temporal bisection task ${ }^{34,35}$ showed that the presentation of foot-shocks or of a cue with a negative valence through its association with foot-shock, produces a drastic disruption of the temporal behavior. Higher levels of stress in our Bad Timers subgroup might have induced the same effect on their timing performances.

It might be argued that the absence of interval-related temporal patterns in respiratory frequency in Bad Timers rats does not mean that these animals were not capable to time intervals, but rather that this ability could not be assessed through respiratory rhythm. Using another index like, for example, heart rate, might have allowed detecting interval timing in these rats ${ }^{36}$. However, as neural activity power in Bad Timers, akin to their respiration rate, was not modulated by interval duration, we feel confident that our classification based on respiratory patterns does indeed reflect the animal's timing ability. Nevertheless, it remains possible that other neural correlates of interval timing might have existed even in animals expressing no temporal behavioral pattern, as described by Tallot et al. ${ }^{37}$.

Some authors have proposed that temporal relations between stimuli are automatically encoded and are the foundation of associative learning ${ }^{8}$. Based on this assumption, it might be expected that animals with poor timing abilities should present lower learning performances than animals with good timing abilities. In the present study Bad Timers and Good Timers showed similar levels of learned odor fear during the $48 \mathrm{~h}$ retention test. However, analyzing more in depth the fear memory strength, by investigating for example if the memory is more 
resistant to extinction or persists longer in Good Timers than in Bad Timers, might reveal learning deficits in Bad Timers that were not detected here.

Importantly, the subdivision of the animals in Good Timers and Bad Timers subgroups based on their respiration temporal patterns, also revealed differences between the two subgroups in the temporal patterns of LFP signal power within the theta band, a result which would potentially not have been detected if data from all animals had been pooled. Specifically, in the Good Timers subgroup, the temporal patterns of theta activity power were modulated by the duration of the odor-shock interval in the four recording sites, and respected scalar property in mPFC and DMS. In contrast, in the Bad Timers subgroup, theta activity power did not change compared to baseline during the odor-shock interval. Concerning activity in the gamma band, although signal power increased in Good Timers and Bad Timers during the interval, only temporal patterns in the mPFC in the Good Timers subgroup were modulated by the duration of the odor-shock interval, but without following scalar property. These data suggest that the learning of interval duration in our paradigm might be supported preferentially by theta oscillatory activity in a network including mainly the mPFC and DMS. Indeed, a dominant model in the field of interval timing is the Striatal Beat Frequency model ${ }^{11,38}$ in which the frontostriatal pathway plays a central role. This model posits that at the start of a significant event, like the arrival of a stimulus signaling the onset of an interval to time, cortical oscillators are synchronized. These cortical neurons project onto the medium spiny neurons of the striatum that detects their coincident activations and compares them to previously stored patterns. In the present study, theta oscillations might facilitate the synchronization of cortical neurons in $\mathrm{mPFC}$ at key moments of the interval to time and enhance the transfer of information along the frontostriatal pathway through increased coherence between mPFC and DMS activities, as recently observed by Tallot et al. ${ }^{37}$.

Our observations are also partly in line with the data reported by Dallérac et al. ${ }^{16}$. In that study, interval timing ability was assessed using a conditioned suppression paradigm, in which a learned aversive cue suppresses ongoing operant behavior consisting in lever pressing for food. Aversive conditioning consisted of a tone paired with a mild electric footshock delivered $30 \mathrm{~s}$ after tone onset. After several weeks of training, rats showed a bellshaped curve of lever-pressing suppression, the maximum conditioned suppression being at a time close to the shock arrival. Furthermore, shifting the tone-shock interval from 30 to 10 s yielded a rapid shift in the peak of suppression. The authors examined oscillatory activity power in DMS and BLA and reported that the power of both theta and gamma oscillations increased during the tone with a maximum that shifted as a result of a change in tone-shock interval duration. Moreover, a significant increase in coherence between these structures was found in the low theta band. This led the authors to propose that learning of the association per se is modulated by the amygdalo-striatal projection through gamma oscillations while the timing component of the association would be driven by theta oscillations.

In the present study, most of the animals were trained successively to the two interval durations. Such a paradigm allows comparing changes within animals, thus discounting between animals' differences, particularly concerning LFP signal power that can be very different between animals due to differences in recording electrode tip position. However, the data obtained during the Shift session might have been partly confounded by the Conditioning session. Indeed, during the Shift session, the animals not only had to learn the new duration but also to forget the previous one. We therefore also compared animals in parallel groups, using a single training session with either $20 \mathrm{~s}$ or $30 \mathrm{~s}$ odor-shock interval duration. The data were similar to those obtained with successive training for respiration temporal patterns, allowing us to assume that these patterns are mostly linked to interval timing per se and not to training levels. Concerning oscillatory activity data, although both parallel and successive training led to temporal patterns modulated by interval duration, these patterns were slightly different between the two conditions particularly concerning gamma activity (see a summary of the data on Table 1, lower part). More specifically, in animals trained in parallel sessions, temporal patterns of gamma activity power follow scalar property in mPFC and DMS while they do not in animals trained in successive sessions. This suggests that gamma activity in these structures keeps a trace of the previously learned interval duration, which could have blurred the $30 \mathrm{~s}$ temporal pattern in our successive paradigm, thus preventing the scalar property to be observed.

In a recent study, Tallot et al. ${ }^{37}$ using a discriminative fear conditioning paradigm where animals had to discriminate between a CS+ (associated with a footshock) and a CS- (never associated with a footshock), showed that early in training, dynamics of neuronal oscillations in an amygdalo-prefronto-striatal network are modified during the CS+ in a manner related to the CS- US time interval. Interestingly these changes occurred while the behavioral response assessed via freezing was similar for CS+ and CS-, indicating high level of fear generalization, thus precluding any causal relationship between time-related modulations in LFPs, and freezing behavior. In the present study, the monitoring of respiration in addition to freezing allowed us to correlate changes in respiratory rate with changes in oscillatory activity power. We found that the temporal patterns of respiratory frequency and theta activity power present striking similarities. Indeed, the time of the respiratory frequency peak (which occurs around $4 \mathrm{~Hz}$ ) was positively correlated with that of the theta activity power peak. In addition, when the duration of the interval was changed, there was a positive correlation between the shift in the respiratory frequency peak time and that of the theta activity power in $\mathrm{mPFC}$. This suggests a potential role for respiratory rhythm in the emergence of theta temporal patterns in the mPFC.

Indeed, several recent studies in the literature have shown that the respiratory rhythm in addition to its impact on olfactory regions (for a review, see Ref. ${ }^{39}$ ), also entrains oscillations in widespread brain regions including those involved in the fear network like the mPFC and amygdala (for a review, see Refs. ${ }^{40,41}$ ). This suggests that the breathing rhythm, akin to slow oscillatory rhythms, could help coordinate neural activity across distant brain regions ${ }^{40,42}$, and potentially modulate cognitive processes. In the present study, it was not possible to analyze the temporal pattern of activity in the $3-5 \mathrm{~Hz}$ delta band due to edge effects surrounding the shock artefact. However, others have found that a $4 \mathrm{~Hz}$ oscillation develops in the mPFC during freezing behavior and that this oscillation was driven by respiration ${ }^{43-45}$. In line with these studies, we recently showed that respiratory frequency and delta activity frequency were highly correlated during freezing ${ }^{29}$. It could be hypothesized that 
the respiration-triggered $4 \mathrm{~Hz}$ oscillation, when it occurs, is particularly well suited to modulate the amplitude of theta and higher frequency oscillations. Importantly we previously reported that the emission of USV, that drastically lowers the respiratory rate down to $0.5-1.5 \mathrm{~Hz}$, disrupted the correlation between respiration and delta rhythm and was associated with a decrease in theta activity power ${ }^{29}$. This could explain why in Bad Timers animals, which present higher USV rates, theta activity power did not increase during the odor-shock interval.

In conclusion, the present work describes for the first time to our knowledge, some of the brain neural dynamics associated with behavioral expression of interval timing at early stages of Pavlovian aversive conditioning. Our study highlights the potential preferential role of theta rhythm in the learning of interval durations, particularly in $\mathrm{mPFC}$ and DMS. In addition, our data suggest the novel idea that the respiratory rhythm might take an active part in the setting of timing-related theta activity dynamics, at least in the mPFC. Whether this role is restricted to the use of an olfactory conditioned stimulus that is sampled through active sniffing or can be observed for another sensory modality, will deserve further investigation. Finally, we were able to show that temporal patterns of theta activity develop concomitantly to temporal patterns of respiration, in a single training session, thus bringing support to the proposal that learning the association between two stimuli co-occurs with the learning of their temporal relationships.

\section{Methods}

Animals. Data were obtained from thirty male Long Evans rats (250-270 g at their arrival, Janvier Labs, France). They were housed individually at $23^{\circ} \mathrm{C}$ and maintained under a $12 \mathrm{~h} \mathrm{light-dark} \mathrm{cycle} \mathrm{(lights} \mathrm{on} \mathrm{from}$ 8:00 a.m. to 8:00 p.m.). Food and water were available ad libitum. All the procedures have been performed in accordance with the European Union guidelines and regulations (Directive 2010/63/EU of the European Parliament and of the Council of the European Union regarding the protection of animals used for scientific purposes), they have been approved by the French Ethical Committee $\mathrm{N}^{\circ} 055$ and the project is referenced by the French Ministry of Research as APAFIS \#10606-2017071309472369v2. Some of the behavioral and electrophysiological data collected on these animals have been used for another paper $^{29}$.

Surgery. Animals were anesthetized with Equithesin, a mixture of chloral hydrate (127 mg/kg, i.p.) and sodium pentobarbital (30 mg/kg, i.p.), and placed in a stereotaxic frame (Narishige, Japan) in a flat skull position. The level of anesthesia was held constant with regular injections of Equithesin throughout the surgery. Monopolar stainless steel recording electrodes $(100 \mu \mathrm{m}$ in diameter) were then stereotaxically implanted in the left hemisphere in the four brain areas: $\mathrm{mPFC}$ (Prelimbic part, AP: $+3.0 \mathrm{~mm}$; L: $+0.8 \mathrm{~mm}$; DV: $-3.5 \mathrm{~mm}$ ), DMS (AP: $+1.2 \mathrm{~mm}, \mathrm{~L}:+2.2 \mathrm{~mm}, \mathrm{DV}:-3.5 \mathrm{~mm})$, PIR (AP: $-1.8, \mathrm{~L}:+5.5 \mathrm{~mm}, \mathrm{DV}:-8 \mathrm{~mm}$ ) and BLA (AP: $-2.8 \mathrm{~mm}$, $\mathrm{L}:+4.9 \mathrm{~mm}, \mathrm{DV}:-7.5 \mathrm{~mm})$. Accurate positioning in the PIR was achieved using the characteristic profiles of evoked field potential induced in the PIR in response to electrical stimulation of the olfactory bulb ${ }^{46}$. For this, a bipolar stimulation electrode (made of two $100-\mu \mathrm{m}$ stainless-steel wires with a tip separation of $500 \mu \mathrm{m}$ ) was lowered transiently in the olfactory bulb to facilitate positioning in the PIR and withdrawn thereafter. A screw inserted in the skull bone above the right parietal lobe served as a reference and ground electrode. The four recording electrodes and the ground electrode were connected to a telemetry transmitter (rodentPACK system, emka TECHNOLOGIES, Paris, France) fixed to the rat's skull surface by dental acrylic cement and anchored with a surgical screw placed in the frontal bone. The animals were allowed to recover for two weeks following surgery.

Experimental apparatus. The apparatus has been described in detail in a previous study ${ }^{24}$. It consisted of a whole body customized plethysmograph (diameter $20 \mathrm{~cm}$, height $30 \mathrm{~cm}$, emka TECHNOLOGIES, France) placed in a sound-attenuating cage (L $60 \mathrm{~cm}, \mathrm{~W} 60 \mathrm{~cm}, \mathrm{H} 70 \mathrm{~cm}, 56 \mathrm{~dB}$ background noise). The plethysmograph was used to measure respiratory parameters in behaving animals. The bottom of the animal chamber was equipped with a shock floor connected to a programmable Coulbourn shocker (Bilaney Consultants $\mathrm{GmbH}$, Düsseldorf, Germany). Three Tygon tubing connected to a programmable custom olfactometer were inserted in the tower on the top of the plethysmograph to deliver air and odorants. Deodorized air flowed constantly through the cage $(2 \mathrm{~L} / \mathrm{min})$. When programmed, an odor (McCormick Pure Peppermint; $2 \mathrm{~L} / \mathrm{min} ; 1: 10$ peppermint vapor to air) was introduced smoothly in the air stream through the switching of a solenoid valve (Fluid Automation Systems, CH-1290 Versoix, Switzerland), thus minimizing its effect on change in pressure. A ventilation pump connected to a port at the bottom of the animal chamber allowed drawing air out of the plethysmograph (at a rate of up to $2 \mathrm{~L} / \mathrm{min}$ ), thus maintaining a constant airflow that did not interact with the animal's breathing pattern. The experimental setup also allowed recording two additional behavioral responses from the animals: freezing behavior was monitored via two video cameras fixed on the walls of the sound-attenuating cage and ultrasonic vocalizations (USV) were recorded via a condenser ultrasound microphone (Avisoft Bioacoustics CM16/CMPA, Berlin, Germany) inserted in a tower adapted to the ceiling of the plethysmograph.

Fear conditioning paradigm (Fig. 1A). After the recovery period, the animals were handled individually and placed in the experimental apparatus for $30 \mathrm{~min}$ per day during 3-4 days before the beginning of the experiments in order to familiarize them with being manipulated and connected to the telemetry transmitter.

For the Conditioning session, the telemetry transmitter was plugged onto the animal's head and the rat was allowed free exploration during the first $4 \mathrm{~min}$, then an odor was introduced into the plethysmograph cage for $20 \mathrm{~s}$ (Group $20 \mathrm{~s}, \mathrm{n}=21$ ), the last second of which overlapped with the delivery of a $0.4 \mathrm{~mA}$ foot-shock $(1 \mathrm{~s})$. The animal received ten odor-shock trials, with an intertrial interval of $4 \mathrm{~min}$. After the last pairing, the transmitter was unplugged, and the animal returned to its home cage. In a small group of animals, the odor-shock interval was set at $30 \mathrm{~s}$ (Group $30 \mathrm{~s}, \mathrm{n}=9$ ), while everything else was kept the same as for Group $20 \mathrm{~s}$. 
The conditioned fear memory was assessed in group $20 \mathrm{~s}$ animals during a Retention test carried out $48 \mathrm{~h}$ after conditioning. For the Retention test, the rat was placed in the experimental cage (equipped with new visual cues and with a plastic floor to avoid contextual fear expression) and allowed a 4-min odor-free period. The CS odor was then presented five times for $20 \mathrm{~s}$ with a 4-min intertrial interval. Two weeks later, these animals were submitted to a Shift session during which the initial $20 \mathrm{~s}$-odor-shock interval was shifted to $30 \mathrm{~s}$. The animal was connected to the telemetry recording system and received ten 30 s-odor-shock trials. After the last pairing the transmitter was unplugged, and the animal returned to its home cage.

Data acquisition and preprocessing. The respiratory signal collected from the plethysmograph was amplified and sent to an acquisition card (MC-1608FS, Measurement Computing, USA; Sampling rate $=1000 \mathrm{~Hz}$ ) for storage and offline analysis. The detection of the respiratory cycles was achieved using an algorithm described in a previous study ${ }^{47}$. This algorithm performs two main operations: signal smoothing for noise reduction, and detection of zero-crossing points to define accurately the inspiration and expiration phase starting points. Momentary respiratory frequency was determined as the inverse of the respiratory cycle (inspiration plus expiration) duration.

The video signals collected through the two cameras were transmitted to a video acquisition card and a homemade acquisition software. Offline, freezing behavior defined as the absence of any visible movement except that due to breathing ${ }^{48}$, was automatically detected using a Labview homemade software and further verified by an experimenter (for a detailed description, see Dupin et al. ${ }^{29}$ ).

For USV recording, the ultrasound microphone was connected to a recording interface (UltraSoundGate 116 $\mathrm{Hb}$, Avisoft Bioacoustics, Berlin, Germany) with the following settings: sampling rate $=214,285 \mathrm{~Hz}$; format $=16$ bit (Wohr et al. ${ }^{25}$ ). Recordings were transferred to Avisoft-SASLab Pro (version 4.2, Avisoft Bioacoustics, Berlin, Germany) and a Fast Fourier Transform (FFT) was conducted. Spectrograms were generated with an FFT-length of 512 points and a time window overlap of $87.5 \%$ (100\% Frame, FlatTop window). These parameters produced a spectrogram at a frequency resolution of $419 \mathrm{~Hz}$ and a time resolution of $0.29 \mathrm{~ms}$. The acoustic signal detection was provided by an automatic whistle tracking algorithm with a threshold of $-20 \mathrm{~dB}$, a minimum duration of $0.01 \mathrm{~s}$ and a hold time of $0.02 \mathrm{~s}$. However, the accuracy of detection was verified trial by trial by an experienced user. The main parameters used in the present study were extracted using Avisoft-SASLab Pro and concerned the duration as well as the start and end time of USV calls. No band pass filter has been applied during USV recording.

Local field potentials (LFP) were collected by telemetry via a four-channel wireless miniature transmitter (<5.2 g, rodentPACK system, emka TECHNOLOGIES). LFP signals were amplified $(1000 \times)$, filtered (between 0.1 and $100 \mathrm{~Hz}$ ), digitized (sampling frequency: $1000 \mathrm{~Hz}$ ) and stored on a computer for offline analysis. The LFP signals were first individually inspected in order to eliminate artifacts due to signal saturation or transient signal loss. The selection was made for each recording site separately and proceeded as follows: when the duration of an artifact exceeded $5 \mathrm{~s}$ over the recording period analyzed, the trial was excluded. When the number of excluded trials exceeded 5 (out of the 10 trials) then the recording site was excluded for this animal. After the elimination of artifacted signals, the number of animals per recording site were the following: BLA, $n=17 ; \mathrm{CPF}, \mathrm{n}=20$; PIR, $\mathrm{n}=20$; DMS, $\mathrm{n}=19$ for Group $20 \mathrm{~s}$; BLA, $\mathrm{n}=6$; CPF, $\mathrm{n}=8$; PIR, $\mathrm{n}=8$; DMS, $\mathrm{n}=9$ for Group $30 \mathrm{~s}$.

Data analysis (Fig. 1B). The different data (respiration, USV, behavior, LFP signals) were synchronized offline via a TTL synchronization signal generated at the beginning of each training session. Once synchronized, the data were analyzed using custom-written scripts in Python.

The temporal course of respiratory frequency and LFP signal power during the $20 \mathrm{~s}$ (or $30 \mathrm{~s}$ ) odor-shock interval was assessed during the Conditioning and Shift sessions as follows. For each animal, the instant respiratory frequency was averaged second by second for each trial of the training session. The power spectral density (PSD) of the LFP signals was calculated using the continuous Morlet wavelet transform ${ }^{49}$. The Morlet wavelet estimated the amplitude of the signal at each time and frequency bin. Two frequency bands were chosen for the subsequent analyses: theta $(5-15 \mathrm{~Hz})$ and gamma $(40-80 \mathrm{~Hz})$, and the mean power in each band was calculated second by second. The resulting values were normalized to baseline defined as the average power over the $20 \mathrm{~s}$ preceding odor onset. The resulting curves of each trial (for respiratory frequency or LFP PSD) were averaged across the trials 2-9 of the session (Trial 1 was not included because the animal had not yet been exposed to the Odor-shock association) for a given animal and then averaged among animals of the same experimental group.

During the Conditioning and Shift sessions, the amount of Freezing and USV emission during the OdorShock intervals was quantified. Freezing rate was expressed as a percentage of the sampled period total duration, averaged across the 9 trials for a given animal and then averaged among animals of the same experimental group. In addition, for each animal, the number of USV emitted during the Odor-Shock intervals throughout the whole session was measured and averaged among animals of the same experimental group.

During the Retention test, the fear memory strength was assessed by measuring the duration of the animal's freezing response before introduction of the first odor, and during each odor presentation. The obtained values were then expressed as a percentage of the sampled period total duration, averaged across the trials for a given animal, and averaged among animals of the same experimental group.

Statistical analysis. All analyses were performed with Systat 13.0 software. For each test, the significance level was set at $\mathrm{p} \leq 0.05$.

In Group $20 \mathrm{~s}$ during training, the per-second temporal curves of respiratory frequency and LFP signal power observed during the Odor-shock interval for the two interval durations were compared using a two-way 
ANOVA with Interval duration (20 s or $30 \mathrm{~s}$ ) and Time (1-18 s) as repeated measures factors. Post-hoc pairwise comparisons were then carried out when allowed by the ANOVA results.

In Group 20 s during Conditioning and Shift sessions, the mean global freezing rate and USV number measured during the Odor-shock interval for the two interval durations were compared using a two-way ANOVA with Subgroup (Good Timers or Bad Timers, see "Results", first paragraph, for Subgroup definition) as an independent factor and Interval duration ( $20 \mathrm{~s}$ or $30 \mathrm{~s}$ ) as a repeated measures factor. Post hoc pairwise comparisons were then carried out when allowed by the ANOVA results.

In Group 20 s during the Retention test, the learned fear response to the odor was analyzed using a two-way ANOVA with Group (Good Timers or Bad Timers) as an independent factor and Period (Pre-odor vs Odor) as a repeated measures factor. Posthoc pairwise comparisons were then carried out when allowed by the ANOVA results.

The per-second temporal curves of respiratory frequency and LFP signal power observed during the first training session in Group $20 \mathrm{~s}$ and Group $30 \mathrm{~s}$ were compared using a two-way ANOVA with Interval duration ( $20 \mathrm{~s}$ or $30 \mathrm{~s})$ as an independent factor and Time (1-18 s) as a repeated measures factor. Posthoc pairwise comparisons were then carried out when allowed by the ANOVA results.

Histology. At the end of the experiment, the animals were injected intraperitoneally with a lethal dose of pentobarbital, their brains were removed, postfixed, cryoprotected in sucrose (20\%). The brains were then sectioned ( $40 \mu \mathrm{m}$ coronal slices) for verification of electrodes tips by light microscopy. Areas targeted by the electrodes in the four implanted brain regions have been reported on schematic brain atlas coronal sections (Supplementary Fig. S6).

Received: 3 August 2020; Accepted: 6 October 2020

Published online: 19 October 2020

\section{References}

1. Roberts, S. Isolation of an internal clock. J. Exp. Psychol. Anim. Behav. Process. 7, 242-268 (1981).

2. Davis, M., Schlesinger, L. S. \& Sorenson, C. A. Temporal specificity of fear conditioning: Effects of different conditioned stimulusunconditioned stimulus intervals on the fear-potentiated startle effect. J. Exp. Psychol. Anim. Behav. Process. 15, 295-310 (1989).

3. Arcediano, F., Escobar, M. \& Miller, R. R. Temporal integration and temporal backward associations in human and nonhuman subjects. Learn. Behav. 31, 242-256 (2003).

4. Boulanger Bertolus, J. et al. Infant rats can learn time intervals before the maturation of the striatum: Evidence from odor fear conditioning. Front. Behav. Neurosci. 8, 176. https://doi.org/10.3389/fnbeh.2014.00176 (2014).

5. Diaz-Mataix, L., Ruiz Martinez, R. C., Schafe, G. E., LeDoux, J. E. \& Doyère, V. Detection of a temporal error triggers reconsolidation of amygdala-dependent memories. Curr. Biol. CB 23, 467-472. https://doi.org/10.1016/j.cub.2013.01.053 (2013).

6. Drew, M. R., Zupan, B., Cooke, A., Couvillon, P. A. \& Balsam, P. D. Temporal control of conditioned responding in goldfish. J. Exp. Psychol. Anim. Behav. Process. 31, 31-39. https://doi.org/10.1037/0097-7403.31.1.31 (2005).

7. Shionoya, K. et al. It's time to fear! Interval timing in odor fear conditioning in rats. Front. Behav. Neurosci. 7, 128. https://doi. org/10.3389/fnbeh.2013.00128 (2013).

8. Balsam, P. D. \& Gallistel, C. R. Temporal maps and informativeness in associative learning. Trends Neurosci. 32, 73-78. https:// doi.org/10.1016/j.tins.2008.10.004 (2009).

9. Tallot, L. \& Doyère, V. Neural encoding of time in the animal brain. Neurosci. Biobehav. Rev. 115, 146-163. https://doi.org/10.1016/j. neubiorev.2019.12.033 (2020).

10. Gibbon, J. \& Church, R. M. Representation of time. Cognition 37, 23-54. https://doi.org/10.1016/0010-0277(90)90017-e (1990).

11. Buhusi, C. V. \& Meck, W. H. What makes us tick? Functional and neural mechanisms of interval timing. Nat. Rev. Neurosci. 6, 755-765. https://doi.org/10.1038/nrn1764 (2005).

12. Coull, J. T., Cheng, R. K. \& Meck, W. H. Neuroanatomical and neurochemical substrates of timing. Neuropsychopharmacology 36, 3-25. https://doi.org/10.1038/npp.2010.113 (2011).

13. Emmons, E. B. et al. Rodent Medial frontal control of temporal processing in the dorsomedial striatum. J. Neurosci. 37, 8718-8733. https://doi.org/10.1523/JNEUROSCI.1376-17.2017 (2017).

14. Emmons, E. B., Kennedy, M., Kim, Y. \& Narayanan, N. S. Corticostriatal stimulation compensates for medial frontal inactivation during interval timing. Sci. Rep. 9, 14371. https://doi.org/10.1038/s41598-019-50975-7 (2019).

15. Diaz-Mataix, L., Tallot, L. \& Doyère, V. The amygdala: A potential player in timing CS-US intervals. Behav. Proc. 101, 112-122. https://doi.org/10.1016/j.beproc.2013.08.007 (2014).

16. Dallérac, G. et al. Updating temporal expectancy of an aversive event engages striatal plasticity under amygdala control. Nat. Commun. 8, 13920. https://doi.org/10.1038/ncomms13920 (2017).

17. Bueti, D. \& Macaluso, E. Auditory temporal expectations modulate activity in visual cortex. NeuroImage 51, 1168-1183. https:// doi.org/10.1016/j.neuroimage.2010.03.023 (2010).

18. Shuler, M. G. \& Bear, M. F. Reward timing in the primary visual cortex. Science 311, 1606-1609. https://doi.org/10.1126/scien ce.1123513 (2006).

19. Hegoburu, C. et al. Differential dynamics of amino acid release in the amygdala and olfactory cortex during odor fear acquisition as revealed with simultaneous high temporal resolution microdialysis. Learn. Memory 16, 687-697. https://doi.org/10.1101/ lm.1584209 (2009).

20. Headley, D. B. \& Weinberger, N. M. Gamma-band activation predicts both associative memory and cortical plasticity. J. Neurosci. 31, 12748-12758. https://doi.org/10.1523/JNEUROSCI.2528-11.2011 (2011).

21. Parker, K. L., Chen, K. H., Kingyon, J. R., Cavanagh, J. F. \& Narayanan, N. S. D1-dependent 4 Hz oscillations and ramping activity in rodent medial frontal cortex during interval timing. J. Neurosci. 34, 16774-16783. https://doi.org/10.1523/JNEUROSCI.277214.2014 (2014).

22. Popescu, A. T., Popa, D. \& Pare, D. Coherent gamma oscillations couple the amygdala and striatum during learning. Nat. Neurosci. 12, 801-807. https://doi.org/10.1038/nn.2305 (2009).

23. Brown, B. L., Hemmes, N. S. \& Cabeza de Vaca, S. Effects of intratrial stimulus change on fixed interval performance: The roles of clock and memory processes. Anim. Learn. Behav. 20, 83-93 (1992). 
24. Hegoburu, C. et al. The RUB Cage: Respiration-ultrasonic vocalizations-behavior acquisition setup for assessing emotional memory in rats. Front. Behav. Neurosci. 5, 25. https://doi.org/10.3389/fnbeh.2011.00025 (2011).

25. Wohr, M., Borta, A. \& Schwarting, R. K. Overt behavior and ultrasonic vocalization in a fear conditioning paradigm: A doseresponse study in the rat. Neurobiol. Learn. Mem. 84, 228-240. https://doi.org/10.1016/j.nlm.2005.07.004 (2005).

26. Arakawa, H. Ethological approach to social isolation effects in behavioral studies of laboratory rodents. Behav. Brain Res. 341, 98-108. https://doi.org/10.1016/j.bbr.2017.12.022 (2018).

27. Manouze, H. et al. Effects of single cage housing on stress, cognitive, and seizure parameters in the rat and mouse pilocarpine models of epilepsy. eNeuro. https://doi.org/10.1523/ENEURO.0179-18.2019 (2019).

28. Frysztak, R. J. \& Neafsey, E. J. The effect of medial frontal cortex lesions on respiration, "freezing," and ultrasonic vocalizations during conditioned emotional responses in rats. Cereb. Cortex 1, 418-425. https://doi.org/10.1093/cercor/1.5.418 (1991).

29. Dupin, M., Garcia, S., Boulanger-Bertolus, J., Buonviso, N. \& Mouly, A. M. New insights from $22-\mathrm{kHz}$ ultrasonic vocalizations to characterize fear responses: Relationship with respiration and brain oscillatory dynamics. eNeuro https://doi.org/10.1523/ENEUR O.0065-19.2019 (2019)

30. Borta, A., Wohr, M. \& Schwarting, R. K. Rat ultrasonic vocalization in aversively motivated situations and the role of individual differences in anxiety-related behavior. Behav. Brain Res. 166, 271-280. https://doi.org/10.1016/j.bbr.2005.08.009 (2006).

31. Brown, B. L., Richer, P. \& Doyère, V. The effect of an intruded event on peak-interval timing in rats: Isolation of a postcue effect. Behav. Proc. 74, 300-310. https://doi.org/10.1016/j.beproc.2006.11.004 (2007).

32. Garces, D. et al. The alteration of emotion regulation precedes the deficits in interval timing in the BACHD rat model for huntington disease. Front. Integr. Neurosci. 12, 14. https://doi.org/10.3389/fnint.2018.00014 (2018).

33. Meck, W. H. \& Macdonald, C. J. Amygdala inactivation reverses fear's ability to impair divided attention and make time stand still. Behav. Neurosci. 121, 707-720. https://doi.org/10.1037/0735-7044.121.4.707 (2007).

34. Faure, A. et al. Modified impact of emotion on temporal discrimination in a transgenic rat model of Huntington disease. Front. Behav. Neurosci. 7, 130. https://doi.org/10.3389/fnbeh.2013.00130 (2013).

35. Kamada, T. \& Hata, T. Basolateral amygdala inactivation eliminates fear-induced underestimation of time in a temporal bisection task. Behav. Brain Res. 356, 227-235. https://doi.org/10.1016/j.bbr.2018.07.027 (2019).

36. Sananes, C. B. \& Campbell, B. A. Role of the central nucleus of the amygdala in olfactory heart rate conditioning. Behav. Neurosci. 103, 519-525 (1989)

37. Tallot, L., Graupner, M., Diaz-Mataix, L. \& Doyère, V. Beyond freezing: Temporal expectancy of an aversive event engages the amygdalo-prefronto-dorsostriatal network. Cereb. Cortex https://doi.org/10.1093/cercor/bhaa100 (2020).

38. Matell, M. S. \& Meck, W. H. Cortico-striatal circuits and interval timing: Coincidence detection of oscillatory processes. Brain Res. Cogn. Brain Res. 21, 139-170. https://doi.org/10.1016/j.cogbrainres.2004.06.012 (2004).

39. Buonviso, N., Amat, C. \& Litaudon, P. Respiratory modulation of olfactory neurons in the rodent brain. Chem. Senses 31, 145-154. https://doi.org/10.1093/chemse/bjj010 (2006).

40. Heck, D. H. et al. Breathing as a fundamental rhythm of brain function. Front. Neural Circuits 10, 115. https://doi.org/10.3389/ fncir.2016.00115 (2016).

41. Tort, A. B. L., Brankack, J. \& Draguhn, A. Respiration-entrained brain rhythms are global but often overlooked. Trends Neurosci. 41, 186-197. https://doi.org/10.1016/j.tins.2018.01.007 (2018).

42. Jensen, O. \& Colgin, L. L. Cross-frequency coupling between neuronal oscillations. Trends Cogn. Sci. 11, 267-269. https://doi. org/10.1016/j.tics.2007.05.003 (2007).

43. Bagur, S. et al. Dissociation of fear initiation and maintenance by breathing-driven prefrontal oscillations. BioRxiv. https://doi. org/10.1101/468264 (2018).

44. Karalis, N. et al. 4-Hz oscillations synchronize prefrontal-amygdala circuits during fear behavior. Nat. Neurosci. 19, 605-612. https ://doi.org/10.1038/nn.4251 (2016).

45. Moberly, A. H. et al. Olfactory inputs modulate respiration-related rhythmic activity in the prefrontal cortex and freezing behavior. Nat. Commun. 9, 1528. https://doi.org/10.1038/s41467-018-03988-1 (2018).

46. Haberly, L. B. Summed potentials evoked in opossum prepyriform cortex. J. Neurophysiol. 36, 775-788 (1973).

47. Roux, S. G. et al. Respiratory cycle as time basis: An improved method for averaging olfactory neural events. J. Neurosci. Methods 152, 173-178. https://doi.org/10.1016/j.jneumeth.2005.09.004 (2006).

48. Blanchard, R. J. \& Blanchard, D. C. Crouching as an index of fear. J. Comp. Physiol. Psychol. 67, 370-375. https://doi.org/10.1037/ h0026779 (1969).

49. Kronland-Martinet, R., Morlet, J. \& Grossmann, A. Analysis of sound patterns through wavelet transforms. Int. J. Pattern Recogn. Artif. Intell. 1, 273-302 (1987).

\section{Acknowledgements}

This work was supported by the Centre National de la Recherche Scientifique, the LABEX CORTEX (ANR-11LABX-0042) of Université de Lyon, within the program "Investissements d'Avenir" (ANR-11-IDEX-0007) operated by the French National Research Agency, and Partner University Funds "Emotion \& Time". We thank Julie Boulanger-Bertolus for valuable discussions of the data and careful reading of the manuscript, Marc Thevenet for technical assistance, and Ounsa Ben-Hellal for taking care of the animals.

\section{Author contributions}

M.D. and A.-M.M. designed the study; M.D. did the experiments; B.M. helped with the design of the experimental setup; M.D., S.G. and A.-M.M. carried out the analyses; M.D. and A.-M.M. wrote the manuscript; V.D. discussed the data, commented and edited the manuscript.

\section{Competing interests}

The authors declare no competing interests.

\section{Additional information}

Supplementary information is available for this paper at https://doi.org/10.1038/s41598-020-74741-2.

Correspondence and requests for materials should be addressed to M.D. or A.-M.M.

Reprints and permissions information is available at www.nature.com/reprints.

Publisher's note Springer Nature remains neutral with regard to jurisdictional claims in published maps and institutional affiliations. 
(c) (i) Open Access This article is licensed under a Creative Commons Attribution 4.0 International cc) License, which permits use, sharing, adaptation, distribution and reproduction in any medium or format, as long as you give appropriate credit to the original author(s) and the source, provide a link to the Creative Commons licence, and indicate if changes were made. The images or other third party material in this article are included in the article's Creative Commons licence, unless indicated otherwise in a credit line to the material. If material is not included in the article's Creative Commons licence and your intended use is not permitted by statutory regulation or exceeds the permitted use, you will need to obtain permission directly from the copyright holder. To view a copy of this licence, visit http://creativecommons.org/licenses/by/4.0/.

(C) The Author(s) 2020 